\title{
General relativity as an extended canonical gauge theory
}

\author{
Jürgen Struckmeier ${ }^{*}$ \\ GSI Helmholtzzentrum für Schwerionenforschung GmbH, Planckstrasse 1, 64291 Darmstadt, Germany \\ and Goethe University, Max-von-Laue-Strasse 1, 60438 Frankfurt am Main, Germany \\ (Received 11 February 2015; published 22 April 2015 in Phys. Rev. D 91, 085030 (2015))
}

\begin{abstract}
It is widely accepted that the fundamental geometrical law of nature should follow from an action principle. The particular subset of transformations of a system's dynamical variables that maintain the form of the action principle comprises the group of canonical transformations. In the context of canonical field theory, the adjective "extended" signifies that not only the fields but also the space-time geometry is subject to transformation. Thus, in order to be physical, the transition to another, possibly noninertial frame of reference must necessarily constitute an extended canonical transformation that defines the general mapping of the connection coefficients, hence the quantities that determine the space-time curvature and torsion of the respective reference frame. The canonical transformation formalism defines simultaneously the transformation rules for the conjugates of the connection coefficients and for the Hamiltonian. As will be shown, this yields unambiguously a particular Hamiltonian that is form-invariant under the canonical transformation of the connection coefficients and thus satisfies the general principle of relativity. This Hamiltonian turns out to be a quadratic function of the curvature tensor. Its Legendre-transformed counterpart then establishes a unique Lagrangian description of the dynamics of space-time that is not postulated but derived from basic principles, namely the action principle and the general principle of relativity. Moreover, the resulting theory satisfies the principle of scale invariance and is renormalizable.
\end{abstract}

PACS numbers: 04.20.Fy, 04.50.Kd, 11.10.Ef, 47.10.Df

\section{INTRODUCTION}

The special principle of relativity states that the fundamental laws of physics must be form-invariant under Lorentz transformations. This can be regarded as to require the description of a system to be form-invariant under a global transformation group, namely the Lorentz group. The generalization to noninertial reference frames is referred to as the general principle of relativity. According to this principle, the description of a system is required to be form-invariant under the corresponding local transformation group, namely the diffeomorphism group that comprises mappings of the local spacetime geometry. In this regard, the transition from the special to the general principle of relativity meets the gauge principle. The latter requires a physical system that happens to be forminvariant under a characteristic global transformation group of the fields to be rendered form-invariant under the corresponding local transformation group, hence the corresponding explicitly space-time dependent transformation group of the fields. For this requirement to be met, a set of "gauge fields" must be added to the system's dynamics that obey a specific inhomogeneous transformation rule. In the case of General Relativity, the local transformation group is constituted by space-time dependent mappings of the local curvature and possibly the local torsion of the reference frames. The "gauge fields" are then given by the connection coefficientsalso referred to as Christoffel symbols for the particular case of a coordinate (holonomic) basis of the reference frame. The connection coefficients also obey a specific inhomogeneous transformation rule relating different reference frames.

The laws which govern the dynamics of classical systems

\footnotetext{
*j.struckmeier@gsi.de
}

can be derived from Hamilton's action principle. In this context, a dynamical system is described by a Lagrangian or its Legendre transform, the Hamiltonian. From the action principle, the dynamics of a classical particle or of a classical field can be derived by integrating the Euler-Lagrange equations or, equivalently, by integrating the canonical equations. The subsequent theory of canonical transformations then isolates exactly the subset of those transformations of the dynamical variables that maintain the form of the action principle-and hence the general form of the canonical equations. As canonical transformations are not restricted to point transformations, the canonical formalism thus establishes the most general path to work out theories that are supposed to be form-invariant under the action of a transformation group of the fields while ensuring the action principle to be maintained. In the extended canonical transformation formalism, the space-time geometry is also subject to transformation. With the space-time then treated as a dynamic variable, a theory that is form-invariant as well under the canonical mapping of the connection coefficients then simultaneously maintains the action principle and satisfies the general principle of relativity.

With this paper, a generalization of the Hamiltonian gauge transformation formalism is reviewed [1] that extends the requirement of form-invariance under a local transformation group of the fields to also demand form-invariance under local transformation of the connection coefficients. Thereby, the well-known formal similarities between non-Abelian gauge theories [2] and general relativity ([3, p 409], [4, p 163]) are encountered. With the transformation rules for the connection coefficients and their respective canonical conjugates being derived from a generating function, it is automatically assured that the extended action principle is preserved, hence that the actual space-time transformation is physical. No additional assumptions need to be incorporated for setting up an amended Hamiltonian that is locally form-invariant on the 
basis of a given globally, hence Lorentz-invariant Hamiltonian. In particular, the connection coefficients are introduced in the most general way by only specifying their transformation properties. No a priori assumptions are incorporated. In particular, it is not assumed that the connection coefficients are symmetric in their lower index pair, hence that a torsion of space-time is excluded [5]. Furthermore, following Palatini's approach [6], the correlation of the connection coefficients with the metric emerges from a canonical equation-or from an Euler-Lagrange equation in the equivalent Lagrangian description - rather than being postulated.

Prior to working out the general local space-time transformation theory in the extended canonical formalism in Sec. $\mathrm{V}$ the formalism of extended Lagrangians and Hamiltonians and their subsequent field equations is presented in Secs. IIand III With the space-time treated as a dynamical variable, the extended Lagrangians and Hamiltonians are defined to also depend on the connection coefficients and their respective conjugates. The general space-time transformation theory in classical vacuum is then based on an extended generating function that defines the mapping of the connection coefficients in the transition from one frame of reference to another. As an extended generating function simultaneously defines the transformation rule for the canonical conjugates of the connection coefficients as well as the transformation law for the extended Hamiltonians, one directly encounters a particular extended Hamiltonian that is form-invariant under the required transformation law of the connection coefficients while maintaining the action principle. The set of canonical field equations following from the obtained gauge-invariant Hamiltonian now establishes a field equation for the Riemann curvature tensor that is no longer postulated, but uniquely emerges from both the action principle and the general principle of relativity.

\section{EXTENDED LAGRANGIANS $\mathcal{L}_{\mathrm{e}}$ IN THE REALM OF CLASSICAL FIELD THEORY}

\section{A. Variational principle, extended set of Euler-Lagrange field equations}

Similar to point dynamics, the Lagrangian formulation of continuum dynamics (see, e.g., [7]) is based on a scalar Lagrange function $\mathcal{L}$ that is supposed to contain the complete information on the given physical system. In a first-order scalar field theory, the Lagrangian $\mathcal{L}$ is defined to depend on $I=1, \ldots, N$ - possibly interacting — scalar fields $\phi_{I}(x)$, on the vector of independent space-time variables $x^{\mu}$, and on the first derivatives of the scalar fields $\phi_{I}$ with respect to the independent variables, i.e., on the covariant vectors (1-forms)

$$
\frac{\partial \phi_{I}}{\partial x^{\nu}} \equiv\left(\frac{\partial \phi_{I}}{\partial x^{0}}, \frac{\partial \phi_{I}}{\partial x^{1}}, \frac{\partial \phi_{I}}{\partial x^{2}}, \frac{\partial \phi_{I}}{\partial x^{3}}\right) .
$$

The Euler-Lagrange field equations are then obtained as the zero of the variation $\delta S$ of the action functional over a spacetime region $R$

$$
S=\int_{R} \mathcal{L}\left(\phi_{I}, \frac{\partial \phi_{I}}{\partial x^{\nu}}, x^{\mu}\right) \mathrm{d}^{4} x, \quad \delta S \stackrel{!}{=} 0
$$

as

$$
\frac{\partial}{\partial x^{j}} \frac{\partial \mathcal{L}}{\partial\left(\frac{\partial \phi_{I}}{\partial x^{j}}\right)}-\frac{\partial \mathcal{L}}{\partial \phi_{I}}=0 .
$$

If the Lagrangian $\mathcal{L}$ is to describe the dynamics of a set of vector fields $a_{I}^{\mu}(x)$ that possibly couple to the scalar fields $\phi_{I}$, then the additional Euler-Lagrange equations take on the similar form

$$
\frac{\partial}{\partial x^{j}} \frac{\partial \mathcal{L}}{\partial\left(\frac{\partial a_{I}^{\mu}}{\partial x^{j}}\right)}-\frac{\partial \mathcal{L}}{\partial a_{I}^{\mu}}=0, \quad \mathcal{L}=\mathcal{L}\left(\phi_{I}, \frac{\partial \phi_{I}}{\partial x^{\nu}}, a_{I}^{\mu}, \frac{\partial a_{I}^{\mu}}{\partial x^{\nu}}, x^{\mu}\right) .
$$

The derivatives of vectors do not transform as tensors. This means that the Euler-Lagrange equations (2) and (3) are not form-invariant under transformations of the space-time metric. Any field equation emerging from Eqs. (2) and (3) that holds a local frame $y$ must finally be rendered a tensor equation in order for the theory described by $\mathcal{L}$ to hold in any reference frame. This is achieved by converting all partial derivatives in the field equations into covariant derivatives.

In analogy to the extended formalism of point mechanics $([8,9])$, the action integral from Eq. (1) can directly be cast into a more general form by decoupling its integration measure from a possibly explicit $x^{\mu}$-dependence of the Lagrangian $\mathcal{L}$

$$
S=\int_{R^{\prime}} \mathcal{L}\left(\phi_{I}, \frac{\partial \phi_{I}}{\partial x^{\nu}}, x^{\mu}\right) \operatorname{det} \Lambda \mathrm{d}^{4} y .
$$

Herein, $\operatorname{det} \Lambda \neq 0$ stands for the determinant of the Jacobi matrix $\Lambda=\left(\Lambda_{\nu^{\prime}}^{\mu}\right)$ that is associated with a regular transformation $x^{\mu} \mapsto y^{\mu}$ of the independent variables and the corresponding transformation $R \mapsto R^{\prime}$ of the integration region

$$
\begin{aligned}
\Lambda & =\left(\Lambda_{\nu^{\prime}}^{\mu}\right)=\left(\begin{array}{ccc}
\frac{\partial x^{0}}{\partial y^{0}} & \cdots & \frac{\partial x^{0}}{\partial y^{3}} \\
\vdots & \ddots & \vdots \\
\frac{\partial x^{3}}{\partial y^{0}} & \cdots & \frac{\partial x^{3}}{\partial y^{3}}
\end{array}\right), \\
\operatorname{det} \Lambda & =\frac{\partial\left(x^{0}, \ldots, x^{3}\right)}{\partial\left(y^{0}, \ldots, y^{3}\right)} \neq 0 \\
\Lambda_{\nu^{\prime}}^{\mu}(y) & =\frac{\partial x^{\mu}(y)}{\partial y^{\nu}}, \quad \Lambda_{\nu}^{\mu^{\prime}}(x)=\frac{\partial y^{\mu}(x)}{\partial x^{\nu}}, \\
\Lambda_{\nu^{\prime}}^{\alpha} \Lambda_{\alpha}^{\mu^{\prime}} & =\Lambda_{\alpha^{\prime}}^{\mu} \Lambda_{\nu}^{\alpha^{\prime}}=\delta_{\nu}^{\mu} .
\end{aligned}
$$

Here, the prime indicates the location of the new independent variables, $y^{\mu}$. As this transformation constitutes a mapping of the space-time metric, the $\Lambda_{\nu^{\prime}}^{\mu}$ are referred to as the spacetime coefficients. The integrand of Eq. (4) can be thought of as defining an extended Lagrangian $\mathcal{L}_{\mathrm{e}}$,

$$
\begin{aligned}
\mathcal{L}_{\mathrm{e}} & \left(\phi_{I}, \frac{\partial \phi_{I}(y)}{\partial y^{\nu}}, x^{\mu}(y), \frac{\partial x^{\mu}(y)}{\partial y^{\nu}}\right) \\
& =\mathcal{L}\left(\phi_{I}, \frac{\partial \phi_{I}(y)}{\partial y^{k}} \frac{\partial y^{k}}{\partial x^{\nu}}, x^{\mu}(y)\right) \operatorname{det} \Lambda .
\end{aligned}
$$

In the language of tensor calculus, the conventional Lagrangian $\mathcal{L}$ represents an absolute scalar whereas the extended Lagrangian $\mathcal{L}_{\mathrm{e}}$ transforms as a relative scalar of weight 
$w=1$ under a mapping of the independent variables, $y^{\mu}$. With this property, $\mathcal{L}_{\mathrm{e}}$ is referred to as a scalar density. Both, $\mathcal{L}$ and $\mathcal{L}_{\mathrm{e}}$ have the dimension of Length $^{-4}$ in natural units $(c=1)$. The now dependent variables $x^{0}, \ldots, x^{3}$ in the argument list of $\mathcal{L}_{\mathrm{e}}$ can be regarded as an extension of the set of fields $\phi_{I}, I=1, \ldots, N$. In other words, the $x^{\mu}(y)$ are treated on equal footing with the fields $\phi_{I}(y)$. In terms of the extended Lagrangian $\mathcal{L}_{\mathrm{e}}$, the action integral over $\mathrm{d}^{4} y$ from Eq. (4) is converted into an integral over an autonomous Lagrangian, hence over a Lagrangian that does not explicitly depend on its independent variables, $y^{\mu}$

$$
S=\int_{R^{\prime}} \mathcal{L}_{\mathrm{e}}\left(\phi_{I}, \frac{\partial \phi_{I}}{\partial y^{\nu}}, x^{\mu}(y), \frac{\partial x^{\mu}}{\partial y^{\nu}}\right) \mathrm{d}^{4} y
$$

As this action integral has exactly the form of the initial one from Eq. (1), the Euler-Lagrange field equations emerging from the variation of Eq. (7) take on form of Eq. (2) (see Appendix C

$$
\frac{\partial}{\partial y^{j}} \frac{\partial \mathcal{L}_{\mathrm{e}}}{\partial\left(\frac{\partial \phi_{I}}{\partial y^{j}}\right)}-\frac{\partial \mathcal{L}_{\mathrm{e}}}{\partial \phi_{I}}=0, \quad \frac{\partial}{\partial y^{j}} \frac{\partial \mathcal{L}_{\mathrm{e}}}{\partial\left(\frac{\partial x^{\mu}}{\partial y^{j}}\right)}-\frac{\partial \mathcal{L}_{\mathrm{e}}}{\partial x^{\mu}}=0 .
$$

An extended Lagrangian $\mathcal{L}_{\mathrm{e}}$ that is correlated to a conventional Lagrangian $\mathcal{L}$ according to Eq. (6) is referred to as a trivial extended Lagrangian. Clearly, multiplying a conventional Lagrangian $\mathcal{L}$ by $\operatorname{det} \Lambda$ and expressing the $x^{\nu}$ derivatives of the fields by means of the chain rule in terms of $y^{\nu}$-derivatives does not add any information. The system's description in terms of a trivial $\mathcal{L}_{\mathrm{e}}$ is thus equivalent to that provided by $\mathcal{L}$.

Yet, a dynamical system that exhibits a dynamical spacetime is generally described by an extended Lagrangian $\mathcal{L}_{\mathrm{e}}$ that does not have a conventional counterpart $\mathcal{L}$. Furthermore, is possible to define extended Lagrangians $\mathcal{L}_{\mathrm{e}}$ that depend in addition on the connection coefficients $\gamma_{\alpha \xi}^{\eta}(x)$ (see Appendix $\mathrm{B}$ and their respective $x^{\nu}$-derivatives that describe the space-time curvature in the $x$ reference frame

$$
S=\int_{R^{\prime}} \mathcal{L}_{\mathrm{e}}\left(\phi_{I}, \frac{\partial \phi_{I}}{\partial y^{\nu}}, \gamma_{\alpha \xi}^{\eta}(x), \frac{\partial \gamma^{\eta}{ }_{\alpha \xi}}{\partial x^{\nu}}, x^{\mu}(y), \frac{\partial x^{\mu}}{\partial y^{\nu}}\right) \mathrm{d}^{4} y
$$

$\delta S \stackrel{!}{=} 0$

This description follows the path of A. Palatini [6, 10], who treated the connection coefficients and the metric as a priori independent quantities. Their correlation then follows from the extended Lagrangian in Eq. (9) by means of the additional Euler-Lagrange equation (see Appendix C)

$$
\frac{\partial}{\partial x^{j}} \frac{\partial \mathcal{L}_{\mathrm{e}}}{\partial\left(\frac{\partial \gamma^{\eta}{ }_{\alpha \xi}}{\partial x^{j}}\right)}-\frac{\partial \mathcal{L}_{\mathrm{e}}}{\partial \gamma^{\eta}{ }_{\alpha \xi}}=0 .
$$

Equations (8) and (10) must be simultaneously fulfilled in order to minimize the action functional (9). This determines uniquely the system's dynamics which includes the dynamics of the space-time geometry.

\section{EXTENDED HAMILTONIANS $\mathcal{H}_{\mathrm{e}}$ IN CLASSICAL FIELD THEORY}

\section{A. Extended canonical field equations}

For a covariant Hamiltonian description, the momentum fields $\pi_{I}^{\nu}$ and $\tilde{\pi}_{I}^{\nu}$ must be defined as the dual quantities of the derivatives of the fields $\phi_{I}$ according to

$$
\pi_{I}^{\nu}(x)=\frac{\partial \mathcal{L}}{\partial\left(\frac{\partial \phi_{I}}{\partial x^{\nu}}\right)}, \quad \tilde{\pi}_{I}^{\nu}(y)=\frac{\partial \mathcal{L}_{\mathrm{e}}}{\partial\left(\frac{\partial \phi_{I}}{\partial y^{\nu}}\right)} .
$$

The momentum fields $\tilde{\pi}_{I}^{\nu}$ emerging from the extended Lagrangian density $\mathcal{L}_{\mathrm{e}}$ transform as

$$
\tilde{\pi}_{I}^{\nu}(y)=\pi_{I}^{j}(x) \frac{\partial y^{\nu}}{\partial x^{j}} \operatorname{det} \Lambda \Leftrightarrow \pi_{I}^{\nu}(x)=\tilde{\pi}_{I}^{j}(y) \frac{\partial x^{\nu}}{\partial y^{j}} \frac{1}{\operatorname{det} \Lambda} .
$$

As indicated by the tilde, the $\tilde{\pi}_{I}^{\nu}=\pi_{I}^{\nu} \operatorname{det} \Lambda$ represent tensor densities of weight $w=1$, whereas the $\pi_{I}^{\nu}$ transform as absolute tensors.

Corresponding to the momentum field $\tilde{\pi}_{I}^{\nu}(y)$ that constitutes the dual counterpart of the Lagrangian dynamical variable $\partial \phi_{I}(y) / \partial y^{\nu}$, the canonical variable $\tilde{t}_{\mu}{ }^{\nu}$ is defined formally as the dual counterpart of $\partial x^{\mu} / \partial y^{\nu}$ and $\tilde{r}_{\eta}^{\alpha \xi \nu}(x)$ as the dual counterpart of $\partial \gamma_{\alpha \xi}^{\eta}(x) / \partial x^{\nu}$. Thus, $\tilde{t}_{\mu}{ }^{\nu}$ and $\tilde{r}_{\eta}{ }^{\alpha \xi \nu}(x)$ follow similarly to Eq. (11) from the respective partial derivative of the extended Lagrangian density $\mathcal{L}_{\mathrm{e}}$ as

$$
\begin{aligned}
\tilde{t}_{\mu}{ }^{\nu} & =-\frac{\partial \mathcal{L}_{\mathrm{e}}}{\partial\left(\frac{\partial x^{\mu}(y)}{\partial y^{\nu}}\right)}=\tilde{t}_{j}{ }^{\nu}(y) \frac{\partial y^{j}}{\partial x^{\mu}}=\tilde{t}_{\mu}{ }^{j}(x) \frac{\partial y^{\nu}}{\partial x^{j}} \\
\tilde{r}_{\eta}{ }^{\alpha \xi \nu}(x) & =\frac{\partial \mathcal{L}_{\mathrm{e}}}{\partial\left(\frac{\partial \gamma^{\eta}{ }_{\alpha \xi}(x)}{\partial x^{\nu}}\right)} .
\end{aligned}
$$

The nontensorial quantity $\gamma_{\alpha \xi}^{\eta}(x)$ must be derived with respect to the $x^{\nu}$ rather than with respect to $y^{\nu}$. Its canonical conjugate $\tilde{r}_{\eta}{ }^{\alpha \xi \nu}(x)$ then also refers to the space-time event $x$. Possible symmetry properties in $\eta, \alpha, \xi$ of $\partial \gamma_{\alpha \xi}^{\eta} / \partial x^{\nu}$ must agree with those of $\tilde{r}_{\eta}{ }^{\alpha \xi \nu}$ in order for both quantities to be actually dual to each other. The Hamiltonian $\mathcal{H}$ and the correlated extended Hamiltonian $\mathcal{H}_{\mathrm{e}}$ can now be defined as the covariant Legendre transform of the Lagrangian $\mathcal{L}$ and the pertaining extended Lagrangian $\mathcal{L}_{\mathrm{e}}$

$$
\begin{aligned}
\mathcal{H}\left(\phi_{I}, \pi_{I}, \gamma, r, x\right) & \\
= & \pi_{J}^{j} \frac{\partial \phi_{J}}{\partial x^{j}}+r_{\eta}{ }^{\alpha \xi j} \frac{\partial \gamma_{\alpha \xi}^{\eta}}{\partial x^{j}} \\
& -\mathcal{L}\left(\phi_{I}, \frac{\partial \phi_{I}}{\partial x^{\nu}}, \gamma_{\alpha \xi}^{\eta}, \frac{\partial \gamma_{\alpha \xi}^{\eta}}{\partial x^{\nu}}, x^{\mu}\right) \\
\mathcal{H}_{\mathrm{e}}\left(\phi_{I}, \tilde{\pi}_{I}, \gamma, \tilde{r}, x, \tilde{t}\right) & \tilde{\pi}_{J}^{j} \frac{\partial \phi_{J}}{\partial y^{j}}+\tilde{r}_{\eta}{ }^{\alpha \xi j} \frac{\partial \gamma_{\alpha \xi}^{\eta}}{\partial x^{j}}-\tilde{t}_{\alpha}{ }^{\beta} \frac{\partial x^{\alpha}}{\partial y^{\beta}} \\
& -\mathcal{L}_{\mathrm{e}}\left(\phi_{I}, \frac{\partial \phi_{I}}{\partial y^{\nu}}, \gamma_{\alpha \xi}^{\eta}, \frac{\partial \gamma_{\alpha \xi}^{\eta}}{\partial x^{\nu}}, x^{\mu}, \frac{\partial x^{\mu}}{\partial y^{\nu}}\right) .
\end{aligned}
$$


The correlation (6) of conventional and extended Lagrangians thus entails a corresponding correlation of conventional and extended Hamiltonians,

$$
\mathcal{H}_{\mathrm{e}}=\mathcal{H} \operatorname{det} \Lambda-\tilde{t}_{\alpha}{ }^{\beta} \frac{\partial x^{\alpha}}{\partial y^{\beta}} .
$$

With this definition of the extended Hamiltonian $\mathcal{H}_{\mathrm{e}}$, one encounters an extended set of the canonical equations. Calculating explicitly the partial derivatives of $\mathcal{H}_{\mathrm{e}}$ from Eq. (14) with respect to all canonical variables yields

$$
\begin{aligned}
\frac{\partial \mathcal{H}_{\mathrm{e}}}{\partial \tilde{\pi}_{I}^{\nu}} & =\frac{\partial \tilde{\pi}_{J}^{j}}{\partial \tilde{\pi}_{I}^{\nu}} \frac{\partial \phi_{J}}{\partial y^{j}}=\delta_{I J} \delta_{\nu}^{j} \frac{\partial \phi_{J}}{\partial y^{j}}=\frac{\partial \phi_{I}}{\partial y^{\nu}} \\
\frac{\partial \mathcal{H}_{\mathrm{e}}}{\partial \tilde{r}_{\eta}^{\alpha \xi^{\nu}}} & =\frac{\partial \tilde{r}_{\eta}^{\alpha \xi j}}{\partial \tilde{r}_{\eta}^{\alpha \xi \nu}} \frac{\partial \gamma_{\alpha \xi}^{\eta}}{\partial x^{j}}=\delta_{\nu}^{j} \frac{\partial \gamma_{\alpha \xi}^{\eta}}{\partial x^{j}}=\frac{\partial \gamma_{\alpha \xi}^{\eta}}{\partial x^{\nu}} \\
\frac{\partial \mathcal{H}_{\mathrm{e}}}{\partial \tilde{t}_{\mu}^{\nu}} & =-\frac{\partial \tilde{t}_{i}^{j}}{\partial \tilde{t}_{\mu}^{\nu}} \frac{\partial x^{i}}{\partial y^{j}}=-\delta_{\nu}^{j} \delta_{i}^{\mu} \frac{\partial x^{i}}{\partial y^{j}}=-\frac{\partial x^{\mu}}{\partial y^{\nu}} \\
\frac{\partial \mathcal{H}_{\mathrm{e}}}{\partial \phi_{I}} & =-\frac{\partial \mathcal{L}_{\mathrm{e}}}{\partial \phi_{I}}=-\frac{\partial}{\partial y^{j}} \frac{\partial \mathcal{L}_{\mathrm{e}}}{\partial\left(\frac{\partial \phi_{I}}{\partial y^{j}}\right)}=-\frac{\partial \tilde{\pi}_{I}^{j}}{\partial y^{j}} \\
\frac{\partial \mathcal{H}_{\mathrm{e}}}{\partial \gamma^{\eta}{ }_{\alpha \xi}} & =-\frac{\partial \mathcal{L}_{\mathrm{e}}}{\partial \gamma^{\eta}{ }_{\alpha \xi}}=-\frac{\partial}{\partial x^{j}} \frac{\partial \mathcal{L}_{\mathrm{e}}}{\partial\left(\frac{\partial \gamma^{\eta}{ }_{\alpha \xi}}{\partial x^{j}}\right)}=-\frac{\partial \tilde{r}_{\eta}^{\alpha \xi j}}{\partial x^{j}} \\
\frac{\partial \mathcal{H}_{\mathrm{e}}}{\partial x^{\mu}} & =-\frac{\partial \mathcal{L}_{\mathrm{e}}}{\partial x^{\mu}}=-\frac{\partial}{\partial y^{j}} \frac{\partial \mathcal{L}_{\mathrm{e}}}{\partial\left(\frac{\partial x^{\mu}}{\partial y^{j}}\right)}=\frac{\partial \tilde{t}_{\mu}^{j}}{\partial y^{j}} .
\end{aligned}
$$

Obviously, an extended Hamiltonian $\mathcal{H}_{\mathrm{e}}$-through its $\phi_{I}$, $\gamma_{\alpha \xi}^{\eta}$, and $x^{\mu}$ dependencies - only determines the divergences $\partial \tilde{\pi}_{I}^{j} / \partial y^{j}, \partial \tilde{r}_{\eta}^{\alpha \xi j} / \partial x^{j}$, and $\partial \tilde{t}_{\mu}^{j} / \partial y^{j}$ but not the individual components $\tilde{\pi}_{I}^{\nu}, \tilde{r}_{\eta}{ }^{\alpha \xi \nu}$, and $\tilde{t}_{\mu}{ }^{\nu}$ of the canonical "momentum" tensor densities. Consequently, the momenta are only determined by the Hamiltonian $\mathcal{H}_{\mathrm{e}}$ up to divergence-free functions.

The action integral from Eq. (9) can be equivalently expressed in terms of the extended Hamiltonian $\mathcal{H}_{\mathrm{e}}$ by applying the Legendre transform (14)

$$
\begin{aligned}
S=\int_{R^{\prime}} & \left(\tilde{\pi}_{J}^{j} \frac{\partial \phi_{J}}{\partial y^{j}}+\tilde{r}_{\eta}^{\alpha \xi j} \frac{\partial \gamma_{\alpha \xi}^{\eta}}{\partial x^{j}}-\tilde{t}_{i}^{j} \frac{\partial x^{i}}{\partial y^{j}}\right. \\
& \left.-\mathcal{H}_{\mathrm{e}}\left(\phi_{I}, \tilde{\pi}_{I}, \gamma, \tilde{r}, x, \tilde{t}\right)\right) \mathrm{d}^{4} y .
\end{aligned}
$$

This representation of the action integral forms the basis on which the extended canonical transformation formalism will be worked out in Sec. IV

In case that the extended Lagrangian describes the dynamics of (covariant) vector fields, $a_{I \mu}(y)$, the canonical momentum fields are to be defined as

$$
\tilde{p}_{I}^{\mu \nu}(y)=\frac{\partial \mathcal{L}_{\mathrm{e}}}{\partial\left(\frac{\partial a_{I \mu}}{\partial y^{\nu}}\right)} .
$$

Similar to Eq. (12), the tensor densities $\tilde{p}_{I}^{\mu \nu}$ then represent the dual quantities of the $y^{\nu}$-derivatives of the vector fields $a_{I \mu}$ and hence the canonical conjugates of the $a_{I \mu}(y)$ in the extended covariant Hamiltonian description.

\section{EXTENDED CANONICAL TRANSFORMATIONS}

\section{A. Generating function of type $\mathcal{F}_{1}$}

In the realm of classical field theory, the condition for defining extended canonical transformations that include mappings $x^{\mu}(y) \mapsto X^{\mu}(y)$ of the respective space-time reference systems $x^{\mu}$ and $X^{\mu}$ and of the connection coefficients $\gamma_{\alpha \xi}^{\eta}(x) \mapsto \Gamma_{\alpha \xi}^{\eta}(X)$ is based on the extended version of the action functional (17). Specifically, the action principle $\delta S \stackrel{!}{=} 0$ is again required to be conserved under the action of the transformation, with $y^{\mu}$ denoting the common independent variables of both reference frames. Requiring the variation of the action functional to be maintained implies the integrand to be determined only up to the divergence of a 4vector function $\mathcal{F}_{1}^{\mu}=\mathcal{F}_{1}^{\mu}\left(\phi_{I}, \Phi_{I}, \gamma, \Gamma, x, X\right)$ on the original and the transformed dynamical "field" variables. The generating function $\mathcal{F}_{1}^{\mu}$ depends on the sets of original fields $\phi_{I}$ and transformed fields $\Phi_{I}$ in conjunction with the connection coefficients $\gamma_{\alpha \xi}^{\eta}(x), \Gamma_{\alpha \xi}^{\eta}(X)$ at the original space-time events $x^{\nu}(y)$ and transformed ones, $X^{\nu}(y)$

$$
\begin{gathered}
\mathcal{L}_{\mathrm{e}}=\mathcal{L}_{\mathrm{e}}^{\prime}+\frac{\partial \mathcal{F}_{1}^{j}}{\partial y^{j}} \Longleftrightarrow \\
\tilde{\pi}_{J}^{j} \frac{\partial \phi_{J}}{\partial y^{j}}+\tilde{r}_{\eta}{ }^{\alpha \xi k} \frac{\partial \gamma^{\eta}{ }_{\alpha \xi}}{\partial x^{k}}-\tilde{t}_{\alpha}{ }^{\beta} \frac{\partial x^{\alpha}}{\partial y^{\beta}}-\mathcal{H}_{\mathrm{e}} \\
=\tilde{\Pi}_{J}^{j} \frac{\partial \Phi_{J}}{\partial y^{j}}+\tilde{R}_{\eta}{ }^{\alpha \xi k} \frac{\partial \Gamma^{\eta}{ }_{\alpha \xi}}{\partial X^{k}}-\tilde{T}_{\alpha}{ }^{\beta} \frac{\partial X^{\alpha}}{\partial y^{\beta}}-\mathcal{H}_{\mathrm{e}}^{\prime}+\frac{\partial \mathcal{F}_{1}^{j}}{\partial y^{j}} .
\end{gathered}
$$

At this point, the tensor densities $\tilde{r}_{\eta}{ }^{\alpha \xi \mu}(x)$ and $\tilde{R}_{\eta}{ }^{\alpha \xi \mu}(X)$ merely denote formally the respective canonical conjugates of the connection coefficients, $\gamma^{\eta}{ }_{\alpha \xi}(x)$ and $\Gamma^{\eta}{ }_{\alpha \xi}(X)$, whose dynamics-and hence whose physical meaning-follows later from the corresponding canonical equation. Since the independent variables $y^{\mu}$ are not transformed, the divergence of the vector function $\mathcal{F}_{1}^{\mu}\left(\phi_{I}, \Phi_{I}, \gamma, \Gamma, x, X\right)$ can be expressed locally as the ordinary divergence

$$
\begin{aligned}
\frac{\partial \mathcal{F}_{1}^{j}}{\partial y^{j}}= & \frac{\partial \mathcal{F}_{1}^{j}}{\partial \phi_{I}} \frac{\partial \phi_{I}}{\partial y^{j}}+\frac{\partial \mathcal{F}_{1}^{j}}{\partial \Phi_{I}} \frac{\partial \Phi_{I}}{\partial y^{j}}+\frac{\partial \mathcal{F}_{1}^{j}}{\partial \gamma^{\eta}} \frac{\partial x^{k}}{\partial y^{j}} \frac{\partial \gamma_{\alpha \xi}^{\eta}}{\partial x^{k}} \\
& +\frac{\partial \mathcal{F}_{1}^{j}}{\partial \Gamma_{\alpha \xi}^{\eta}} \frac{\partial X^{k}}{\partial y^{j}} \frac{\partial \Gamma_{\alpha \xi}^{\eta}}{\partial X^{k}}+\frac{\partial \mathcal{F}_{1}^{j}}{\partial x^{i}} \frac{\partial x^{i}}{\partial y^{j}}+\frac{\partial \mathcal{F}_{1}^{j}}{\partial X^{i}} \frac{\partial X^{i}}{\partial y^{j}} .
\end{aligned}
$$

Comparing the coefficients of Eqs. (19) and (20), the extended local coordinate representation of the transformation rules induced by the extended generating function $\mathcal{F}_{1}^{\mu}(y)$ are

$$
\begin{aligned}
\tilde{\pi}_{I}^{\mu} & =\frac{\partial \mathcal{F}_{1}^{\mu}}{\partial \phi_{I}}, \quad \tilde{\Pi}_{I}^{\mu}=-\frac{\partial \mathcal{F}_{1}^{\mu}}{\partial \Phi_{I}}, \quad \tilde{r}_{\eta}{ }^{\alpha \xi \mu}=\frac{\partial \mathcal{F}_{1}^{j}}{\partial \gamma^{\eta}{ }_{\alpha \xi}} \frac{\partial x^{\mu}}{\partial y^{j}} \\
\tilde{R}_{\eta}{ }^{\alpha \xi \mu} & =-\frac{\partial \mathcal{F}_{1}^{j}}{\partial \Gamma^{\eta}{ }_{\alpha \xi}} \frac{\partial X^{\mu}}{\partial y^{j}}, \quad \tilde{t}_{\nu}{ }^{\mu}=-\frac{\partial \mathcal{F}_{1}^{\mu}}{\partial x^{\nu}}, \quad \tilde{T}_{\nu}{ }^{\mu}=\frac{\partial \mathcal{F}_{1}^{\mu}}{\partial X^{\nu}} \\
\mathcal{H}_{\mathrm{e}}^{\prime} & =\mathcal{H}_{\mathrm{e}}, \mathcal{H}^{\prime} \operatorname{det} \Lambda^{\prime}-\tilde{T}_{\alpha}{ }^{\beta} \frac{\partial X^{\alpha}}{\partial y^{\beta}}=\mathcal{H} \operatorname{det} \Lambda-\tilde{t}_{\alpha}{ }^{\beta} \frac{\partial x^{\alpha}}{\partial y^{\beta}} .
\end{aligned}
$$


Note that the nontensor quantities $\gamma_{\alpha \xi}^{\eta}(x)$ must always refer to the original reference system, $x$, and, correspondingly, $\Gamma_{\alpha \xi}^{\eta}(X)$ to the transformed reference system, $X$. The reference systems of their local canonical conjugates, hence the tensor densities $\tilde{r}_{\eta}^{\alpha \xi \mu}(x)$ and $\tilde{R}_{\eta}{ }^{\alpha \xi \mu}(X)$ are defined accordingly. The indices of $\tilde{t}_{\nu}{ }^{\mu}$ and $\tilde{T}_{\nu}{ }^{\mu}$ in Eq. (21) are related to different reference systems. The upper index of $\tilde{t}_{\nu}{ }^{\mu}$ and $\tilde{T}_{\nu}{ }^{\mu}$ pertains to the reference system $y$, whereas the lower index refers to the reference systems $x$ and $X$, respectively. In order to attribute these quantities a definite space-time location, hence to convert them into regular tensors, the transformations follow as

$$
\begin{gathered}
\tilde{\theta}_{\nu}{ }^{\mu}(x)=\tilde{t}_{\nu}{ }^{j} \frac{\partial x^{\mu}}{\partial y^{j}}=-\frac{\partial \mathcal{F}_{1}^{j}}{\partial x^{\nu}} \frac{\partial x^{\mu}}{\partial y^{j}} \\
\tilde{\Theta}_{\nu}{ }^{\mu}(X)=\tilde{T}_{\nu}{ }^{j} \frac{\partial X^{\mu}}{\partial y^{j}}=\frac{\partial \mathcal{F}_{1}^{j}}{\partial X^{\nu}} \frac{\partial X^{\mu}}{\partial y^{j}},
\end{gathered}
$$

in agreement with the definition of $\tilde{t}_{\nu}{ }^{\mu}$ as the dual quantity of $\partial x^{\nu} / \partial y^{\mu}$ from Eq. (13). As shown in Appendix A, the tensor $\theta_{\nu}{ }^{\mu}(x)$ represents the canonical energy-momentum tensor of the original system if the dynamical system is described by a trivial extended Lagrangian $\mathcal{L}_{\mathrm{e}}$ or its corresponding Legendre-transform that defines a trivial extended Hamiltonian, $\mathcal{H}_{\mathrm{e}}$.

According to Eqs. (21), the value of an extended Hamiltonian $\mathcal{H}_{\mathrm{e}}$ is conserved under extended canonical transformations. Hence, the transformed extended Hamiltonian density $\mathcal{H}_{\mathrm{e}}^{\prime}\left(\Phi_{I}, \tilde{\Pi}{ }_{I}, \Gamma, \tilde{R}, X, \tilde{T}\right)$ is obtained by expressing the original extended Hamiltonian $\mathcal{H}_{\mathrm{e}}\left(\phi_{I}, \tilde{\pi}_{I}, \gamma, \tilde{r}, x, \tilde{t}\right)$ in terms of the transformed fields $\Phi_{I}, \tilde{\Pi}_{I}^{\mu}$, the transformed $\Gamma_{\alpha \xi}^{\eta}, \tilde{R}_{\eta}{ }^{\alpha \xi \mu}$ and the transformed space-time location $X^{\nu}$, and its canonical conjugate, $\tilde{T}_{\nu}{ }^{\mu}$.

\section{B. Generating function of type $\mathcal{F}_{2}$}

The generating function of an extended canonical transformation can alternatively be expressed in terms of a function $\mathcal{F}_{2}^{\mu}$ of the original fields $\phi_{I}$, the connection coefficients of the original system, $\gamma_{\alpha \xi}^{\eta}(x)$, and the original space-time coordinates, $x^{\mu}$ in conjunction with the new conjugate fields $\tilde{\Pi}_{I}^{\mu}$, the $\tilde{R}_{\eta}{ }^{\alpha \xi \mu}$ as the duals to $\Gamma^{\eta}{ }_{\alpha \xi}(X)$, and the $\tilde{T}_{\nu}{ }^{\mu}$ as the duals to $-\partial X^{\nu} / \partial y^{\mu}$. In order to derive the pertaining transformation rules, the following extended Legendre transformation is performed

$$
\begin{aligned}
& \mathcal{F}_{2}^{\mu}\left(\phi_{I}, \tilde{\Pi}_{I}, \gamma, \tilde{R}, x, \tilde{T}\right) \\
& =\mathcal{F}_{1}^{\mu}\left(\phi_{I}, \Phi_{I}, \gamma, \Gamma, x, X\right)+\Phi_{I} \tilde{\Pi}_{I}^{\mu} \\
& \quad+\Gamma_{\alpha \xi}^{\eta} \tilde{R}_{\eta}{ }^{\alpha \xi j} \frac{\partial y^{\mu}}{\partial X^{j}}-X^{j} \tilde{T}_{j}{ }^{\mu} .
\end{aligned}
$$

The resulting transformation rules are

$$
\begin{gathered}
\tilde{\pi}_{I}^{\mu}=\frac{\partial \mathcal{F}_{2}^{\mu}}{\partial \phi_{I}}, \quad \Phi_{I} \delta_{\nu}^{\mu}=\frac{\partial \mathcal{F}_{2}^{\mu}}{\partial \tilde{\Pi}_{I}^{\nu}}, \quad \tilde{r}_{\eta}{ }^{\alpha \xi \mu}=\frac{\partial \mathcal{F}_{2}^{j}}{\partial \gamma^{\eta}{ }_{\alpha \xi}} \frac{\partial x^{\mu}}{\partial y^{j}} \\
\Gamma_{\alpha \xi}^{\eta} \delta_{\nu}^{\mu}=\frac{\partial \mathcal{F}_{2}^{\mu}}{\partial \tilde{R}_{\eta}{ }^{\alpha \xi j}} \frac{\partial X^{j}}{\partial y^{\nu}}, \quad \tilde{t}_{\nu}{ }^{\mu}=-\frac{\partial \mathcal{F}_{2}^{\mu}}{\partial x^{\nu}}, \quad X^{\alpha} \delta_{\nu}^{\mu}=-\frac{\partial \mathcal{F}_{2}^{\mu}}{\partial \tilde{T}_{\alpha}{ }^{\nu}}
\end{gathered}
$$

$$
\mathcal{H}_{\mathrm{e}}^{\prime}=\mathcal{H}_{\mathrm{e}}, \mathcal{H}^{\prime} \operatorname{det} \Lambda^{\prime}-\tilde{T}_{\alpha}{ }^{\beta} \frac{\partial X^{\alpha}}{\partial y^{\beta}}=\mathcal{H} \operatorname{det} \Lambda-\tilde{t}_{\alpha}{ }^{\beta} \frac{\partial x^{\alpha}}{\partial y^{\beta}}
$$

These transformation rules are equivalent to the set of Eqs. (21) if the Legendre transformation (22) is nonsingular, hence if the determinant of the Hesse matrix of $\mathcal{F}_{1}$ is nonzero.

\section{GENERAL SPACE-TIME TRANSFORMATION IN VACUUM}

Given a classical vacuum system, i.e., a system with no fields. An extended canonical transformation that maps the space-time geometry from a space-time location $x$ to $X$ under the transformation law of the connection coefficients $\gamma_{\alpha \xi}^{\eta}(x) \mapsto \Gamma_{\alpha \xi}^{\eta}(X)$ in a coordinate basis (see Appendix B) is generated by

$$
\begin{aligned}
\mathcal{F}_{2}^{\mu}(\gamma, \tilde{R}, x, \tilde{T}) & =-\tilde{T}_{\alpha}{ }^{\mu} h^{\alpha}(x)+\tilde{R}_{\eta}{ }^{\alpha \xi \lambda} \frac{\partial y^{\mu}}{\partial X^{\lambda}} \\
\times & \left(\gamma^{k}{ }_{i j} \frac{\partial X^{\eta}}{\partial x^{k}} \frac{\partial x^{i}}{\partial X^{\alpha}} \frac{\partial x^{j}}{\partial X^{\xi}}+\frac{\partial X^{\eta}}{\partial x^{k}} \frac{\partial^{2} x^{k}}{\partial X^{\alpha} \partial X^{\xi}}\right)
\end{aligned}
$$

In this definition of an extended generating function of type $\mathcal{F}_{2}^{\mu}(y)$, the tensor density components $\tilde{R}_{\eta}{ }^{\alpha \xi \lambda}(X) \partial y^{\mu} / \partial X^{\lambda}$ denote formally the canonical conjugates of the connection coefficients $\Gamma_{\alpha \xi}^{\eta}(X)$ of the transformed system and hence the dual quantities to the $y^{\mu}$-derivatives of the $\Gamma_{\alpha \xi}^{\eta}(X)$. The $\gamma_{\alpha \xi}^{\eta}(x)$ stand for the connection coefficients of the original system. The tensor density components $\tilde{R}_{\eta}{ }^{\alpha \xi \mu}(X) \equiv$ $R_{\eta}{ }^{\alpha \xi \mu}(X) \operatorname{det} \Lambda^{\prime}$ then represent the dual quantities of the $X^{\mu}$-derivatives of the transformed connection coefficients $\Gamma_{\alpha \xi}^{\eta}(X)$

$$
\tilde{R}_{\eta}{ }^{\alpha \xi \lambda}(X) \frac{\partial y^{\mu}}{\partial X^{\lambda}} \frac{\partial \Gamma^{\eta}{ }_{\alpha \xi}(X)}{\partial y^{\mu}}=R_{\eta}{ }^{\alpha \xi \lambda}(X) \frac{\partial \Gamma^{\eta}{ }_{\alpha \xi}(X)}{\partial X^{\lambda}} \operatorname{det} \Lambda^{\prime} .
$$

Likewise, the tensor density components $\tilde{r}_{\eta}{ }^{\alpha \xi \mu}(x) \equiv$ $r_{\eta}{ }^{\alpha \xi \mu}(x) \operatorname{det} \Lambda$ denote the dual quantities of the $x^{\mu}$ derivatives of the connection coefficients $\gamma^{\eta}{ }_{\alpha \xi}(x)$ of the original system. No predication with respect to the physical meaning of $r_{\eta}{ }^{\alpha \xi \mu}$ and $R_{\eta}{ }^{\alpha \xi \mu}$ is made at this point.

The particular generating function 24 entails the following transformation rules according to the general rules from Eqs. (23)

$$
\begin{aligned}
\Gamma_{\alpha \xi}^{\eta} \delta_{\nu}^{\mu} & =\frac{\partial \mathcal{F}_{2}^{\mu}}{\partial \tilde{R}_{\eta}^{\alpha \xi j}} \frac{\partial X^{j}}{\partial y^{\nu}} \\
& =\delta_{\nu}^{\mu}\left(\gamma_{i j}^{k} \frac{\partial X^{\eta}}{\partial x^{k}} \frac{\partial x^{i}}{\partial X^{\alpha}} \frac{\partial x^{j}}{\partial X^{\xi}}+\frac{\partial X^{\eta}}{\partial x^{k}} \frac{\partial^{2} x^{k}}{\partial X^{\alpha} \partial X^{\xi}}\right) \\
\tilde{r}_{k}{ }^{i j \mu} & =\frac{\partial \mathcal{F}_{2}^{\kappa}}{\partial \gamma_{i j}^{k}} \frac{\partial x^{\mu}}{\partial y^{\kappa}}=\tilde{R}_{\eta}{ }^{\alpha \xi \lambda} \frac{\partial X^{\eta}}{\partial x^{k}} \frac{\partial x^{i}}{\partial X^{\alpha}} \frac{\partial x^{j}}{\partial X^{\xi}} \frac{\partial x^{\mu}}{\partial X^{\lambda}} \\
X^{\alpha} \delta_{\nu}^{\mu} & =-\frac{\partial \mathcal{F}_{2}^{\mu}}{\partial \tilde{T}_{\alpha}{ }^{\nu}}=\delta_{\nu}^{\mu} h^{\alpha}(x)
\end{aligned}
$$




$$
\begin{aligned}
\tilde{t}_{\nu}{ }^{\mu}= & -\frac{\partial \mathcal{F}_{2}^{\mu}}{\partial x^{\nu}} \\
= & \tilde{T}_{\alpha}{ }^{\mu} \frac{\partial h^{\alpha}(x)}{\partial x^{\nu}}-\tilde{R}_{\eta}{ }^{\alpha \xi \lambda} \frac{\partial y^{\mu}}{\partial X^{\lambda}} \\
& \times\left[\gamma^{k}{ }_{i j} \frac{\partial}{\partial x^{\nu}}\left(\frac{\partial X^{\eta}}{\partial x^{k}} \frac{\partial x^{i}}{\partial X^{\alpha}} \frac{\partial x^{j}}{\partial X^{\xi}}\right)\right. \\
& \left.+\frac{\partial}{\partial x^{\nu}}\left(\frac{\partial X^{\eta}}{\partial x^{k}} \frac{\partial^{2} x^{k}}{\partial X^{\alpha} \partial X^{\xi}}\right)\right]
\end{aligned}
$$

hence

$$
\begin{aligned}
\Gamma_{\alpha \xi}^{\eta}(X)= & \gamma^{k}{ }_{i j}(x) \frac{\partial X^{\eta}}{\partial x^{k}} \frac{\partial x^{i}}{\partial X^{\alpha}} \frac{\partial x^{j}}{\partial X^{\xi}}+\frac{\partial X^{\eta}}{\partial x^{k}} \frac{\partial^{2} x^{k}}{\partial X^{\alpha} \partial X^{\xi}} \\
\tilde{r}_{k}{ }^{i j \mu}(x)= & \tilde{R}_{\eta}{ }^{\alpha \xi \lambda}(X) \frac{\partial X^{\eta}}{\partial x^{k}} \frac{\partial x^{i}}{\partial X^{\alpha}} \frac{\partial x^{j}}{\partial X^{\xi}} \frac{\partial x^{\mu}}{\partial X^{\lambda}} \\
X^{\mu}= & h^{\mu}(x) \\
\tilde{t}_{\nu}{ }^{\mu}= & \tilde{T}_{\alpha}{ }^{\mu} \frac{\partial X^{\alpha}}{\partial x^{\nu}}-\tilde{R}_{\eta}{ }^{\alpha \xi \lambda} \frac{\partial y^{\mu}}{\partial X^{\lambda}} \\
& \times\left[\gamma^{k}{ }_{i j} \frac{\partial}{\partial x^{\nu}}\left(\frac{\partial X^{\eta}}{\partial x^{k}} \frac{\partial x^{i}}{\partial X^{\alpha}} \frac{\partial x^{j}}{\partial X^{\xi}}\right)\right. \\
& \left.+\frac{\partial}{\partial x^{\nu}}\left(\frac{\partial X^{\eta}}{\partial x^{k}} \frac{\partial^{2} x^{k}}{\partial X^{\alpha} \partial X^{\xi}}\right)\right] .
\end{aligned}
$$

According to Eqs. (23), the last rule yields the transformation rule for the conventional Hamiltonians via

$$
\begin{aligned}
& \tilde{T}_{\alpha}{ }^{\beta} \frac{\partial X^{\alpha}}{\partial y^{\beta}}-\tilde{t}_{\alpha}{ }^{\beta} \frac{\partial x^{\alpha}}{\partial y^{\beta}} \\
&=\mathcal{H}^{\prime} \operatorname{det} \Lambda^{\prime}-\mathcal{H} \operatorname{det} \Lambda \\
&=\tilde{R}_{\eta}{ }^{\alpha \xi \lambda} \frac{\partial x^{\nu}}{\partial X^{\lambda}}\left[\gamma^{k}{ }^{k} \frac{\partial}{\partial x^{\nu}}\left(\frac{\partial X^{\eta}}{\partial x^{k}} \frac{\partial x^{i}}{\partial X^{\alpha}} \frac{\partial x^{j}}{\partial X^{\xi}}\right)\right. \\
&\left.\quad+\frac{\partial}{\partial x^{\nu}}\left(\frac{\partial X^{\eta}}{\partial x^{k}} \frac{\partial^{2} x^{k}}{\partial X^{\alpha} \partial X^{\xi}}\right)\right] .
\end{aligned}
$$

Yet, what is actually desired is the transformation rule for the Hamiltonians as expressed in terms of their proper dynamical variables $\gamma, r$ and $\Gamma, R$, respectively. This requires to express all derivatives of the functions $x^{\mu}(y)$ and $X^{\mu}(y)$ in (26) in terms of the original and transformed connection coefficients $\gamma_{\alpha \xi}^{\eta}(x)$ and $\Gamma_{\alpha \xi}^{\eta}(X)$ and their conjugates, $\tilde{r}_{\eta}{ }^{\alpha \xi \mu}$ and $\tilde{R}_{\eta}{ }^{\alpha \xi \mu}$, by making use of the respective canonical transformation rules (25). This calculation is worked out explicitly in Appendix D Remarkably, the transformation rule (26) can indeed completely and symmetrically be expressed in terms of the canonical variables of the original and the transformed system as

$$
\begin{aligned}
\tilde{T}_{\alpha}{ }^{\beta} & \frac{\partial X^{\alpha}}{\partial y^{\beta}}-\tilde{t}_{\alpha}{ }^{\beta} \frac{\partial x^{\alpha}}{\partial y^{\beta}} \\
= & \frac{1}{2} \tilde{R}_{\eta}{ }^{\alpha \xi \mu}\left(\frac{\partial \Gamma^{\eta}{ }_{\alpha \xi}}{\partial X^{\mu}}+\frac{\partial \Gamma^{\eta}{ }_{\alpha \mu}}{\partial X^{\xi}}-\Gamma_{\alpha \xi}^{k} \Gamma_{k \mu}^{\eta}+\Gamma_{\alpha \mu}^{k} \Gamma_{k \xi}^{\eta}\right) \\
& \quad-\frac{1}{2} \tilde{r}_{\eta}{ }^{\alpha \xi \mu}\left(\frac{\partial \gamma_{\alpha \xi}^{\eta}}{\partial x^{\mu}}+\frac{\partial \gamma^{\eta}{ }_{\alpha \mu}}{\partial x^{\xi}}-\gamma_{\alpha \xi}^{k} \gamma_{k \mu}^{\eta}+\gamma_{\alpha \mu}^{k} \gamma_{k \xi}^{\eta}\right) .
\end{aligned}
$$

Gathering original and transformed dynamical variables on ei- ther side of the equation yields

$$
\begin{gathered}
\frac{1}{2} \tilde{R}_{\eta}{ }^{\alpha \xi \mu}\left(\frac{\partial \Gamma^{\eta}{ }_{\alpha \xi}}{\partial X^{\mu}}+\frac{\partial \Gamma_{\alpha \mu}^{\eta}}{\partial X^{\xi}}-\Gamma_{\alpha \xi}^{k} \Gamma_{k \mu}^{\eta}+\Gamma_{\alpha \mu}^{k} \Gamma_{k \xi}^{\eta}\right) \\
-\tilde{T}_{\alpha}{ }^{\beta} \frac{\partial X^{\alpha}}{\partial y^{\beta}} \\
=\frac{1}{2} \tilde{r}_{\eta}{ }^{\alpha \xi \mu}\left(\frac{\partial \gamma^{\eta}{ }_{\alpha \xi}}{\partial x^{\mu}}+\frac{\partial \gamma^{\eta}{ }_{\alpha \mu}}{\partial x^{\xi}}-\gamma_{\alpha \xi}^{k} \gamma_{k \mu}^{\eta}+\gamma_{\alpha \mu}^{k} \gamma_{k \xi}^{\eta}\right) \\
-\tilde{t}_{\alpha}{ }^{\beta} \frac{\partial x^{\alpha}}{\partial y^{\beta}} .
\end{gathered}
$$

The left- and right-hand sides of this equation can be regarded as extended Hamiltonians $\mathcal{H}_{\mathrm{e}}^{\prime}$ and $\mathcal{H}_{\mathrm{e}}$, respectively, which satisfy the required transformation rule $\mathcal{H}_{\mathrm{e}}^{\prime}=\mathcal{H}_{\mathrm{e}}$ from Eqs. (23). Obviously, the Hamiltonians not only retain their values but are furthermore form-invariant under the extended canonical transformation generated by Eq. (24).

In order for the canonical equations following from the Hamiltonians $\mathcal{H}_{\mathrm{e}}$ and $\mathcal{H}_{\mathrm{e}}^{\prime}$ to be compatible with the canonical transformation rules (25), the above form-invariant Hamiltonians must be amended, by "free field" Hamiltonians

$$
\begin{aligned}
& \mathcal{H}_{\mathrm{e}, \mathrm{dyn}}^{\prime}=-\frac{1}{4} R_{\eta}{ }^{\alpha \xi \mu} R^{\eta}{ }_{\alpha \xi \mu} \operatorname{det} \Lambda^{\prime}, \\
& \mathcal{H}_{\mathrm{e}, \mathrm{dyn}}=-\frac{1}{4} r_{\eta}{ }^{\alpha \xi \mu} r^{\eta}{ }_{\alpha \xi \mu} \operatorname{det} \Lambda .
\end{aligned}
$$

Clearly, $\mathcal{H}_{\mathrm{e}, \mathrm{dyn}}^{\prime}=\mathcal{H}_{\mathrm{e}, \mathrm{dyn}}$ must hold under the rules (25) in order for the final extended Hamiltonians to maintain the required transformation rule $\mathcal{H}_{\mathrm{e}}^{\prime}=\mathcal{H}_{\mathrm{e}}$. This is ensured if $\operatorname{det} \Lambda^{\prime}=\operatorname{det} \Lambda$, hence if

$$
\frac{\partial\left(X^{0}, \ldots, X^{3}\right)}{\partial\left(x^{0}, \ldots, x^{3}\right)}=\frac{\partial\left(h^{0}(x), \ldots, h^{3}(x)\right)}{\partial\left(x^{0}, \ldots, x^{3}\right)}=1 .
$$

Thus, the by now arbitrary function $h^{\alpha}(x)$ in the generating function (24) must satisfy Eq. (28). The final form-invariant extended Hamiltonian that is compatible with the extended set of canonical transformation rules generated by (24) now writes for the $x$ reference frame

$$
\begin{aligned}
& \mathcal{H}_{\mathrm{e}}(\tilde{r}, \gamma, \tilde{t})=-\tilde{t}_{\alpha}{ }^{\beta} \frac{\partial x^{\alpha}}{\partial y^{\beta}}-\frac{1}{4} \tilde{r}_{\eta}{ }^{\alpha \xi \mu} r_{\alpha \xi \mu}^{\eta} \\
& \quad+\frac{1}{2} \tilde{r}_{\eta}{ }^{\alpha \xi \mu}\left(\frac{\partial \gamma_{\alpha \mu}^{\eta}}{\partial x^{\xi}}+\frac{\partial \gamma_{\alpha \xi}^{\eta}}{\partial x^{\mu}}+\gamma_{\alpha \mu}^{k} \gamma_{k \xi}^{\eta}-\gamma_{\alpha \xi}^{k} \gamma_{k \mu}^{\eta}\right) .
\end{aligned}
$$

\section{CANONICAL EQUATIONS}

The canonical equation for the connection coefficients follows as

$$
\begin{aligned}
\frac{\partial \gamma^{\eta}{ }_{\alpha \xi}}{\partial x^{\mu}}= & \frac{\partial \mathcal{H}_{\mathrm{e}}}{\partial \tilde{r}_{\eta}{ }^{\alpha \xi \mu}} \\
= & -\frac{1}{2} r^{\eta}{ }_{\alpha \xi \mu} \\
& +\frac{1}{2}\left(\frac{\partial \gamma^{\eta}{ }_{\alpha \mu}}{\partial x^{\xi}}+\frac{\partial \gamma_{\alpha \xi}^{\eta}}{\partial x^{\mu}}+\gamma^{k}{ }_{\alpha \mu} \gamma_{k \xi}^{\eta}-\gamma_{\alpha \xi}^{k} \gamma_{k \mu}^{\eta}\right) .
\end{aligned}
$$


Solved for $r_{\alpha \xi \mu}^{\eta}$ one finds

$$
r_{\alpha \xi \mu}^{\eta}=\frac{\partial \gamma_{\alpha \mu}^{\eta}}{\partial x^{\xi}}-\frac{\partial \gamma_{\alpha \xi}^{\eta}}{\partial x^{\mu}}+\gamma_{\alpha \mu}^{k} \gamma_{k \xi}^{\eta}-\gamma_{\alpha \xi}^{k} \gamma_{k \mu}^{\eta} .
$$

One thus encounters exactly the representation of the Riemann curvature tensor in terms of the connection coefficients and their derivatives. The quantity $\tilde{R}_{\eta}{ }^{\alpha \xi \lambda}(X) \partial y^{\mu} / \partial X^{\lambda}$ was formally introduced in the generating function (24) as the canonical conjugate of the connection coefficient $\Gamma^{\eta}{ }_{\alpha \xi}(X)$ in order to yield the required transformation law for $\gamma^{\eta}{ }_{\alpha \xi}(x)$. With Eq. (30), the quantity $r_{\eta}{ }^{\alpha \xi \mu}$ is now attributed a physical meaning. It is manifestly skew-symmetric in the indices $\xi$ and $\mu$. With Eq. (30) being a tensor equation, the second canonical transformation (25) rule is satisfied, which requires $r^{\eta}{ }_{\alpha \xi \mu}$ to transform as a tensor.

The divergence of $\tilde{r}_{\eta}{ }^{\alpha \xi \mu}$ follows from the derivative of $\mathcal{H}_{\mathrm{e}}$ with respect to $\gamma_{\alpha \xi}^{\eta}$

$$
\left(\tilde{r}_{\eta}{ }^{\alpha \xi \beta}\right)_{, \beta} \equiv \frac{\partial \tilde{r}_{\eta}^{\alpha \xi \beta}}{\partial x^{\beta}}=-\frac{\partial \mathcal{H}_{\mathrm{e}}}{\partial \gamma_{\alpha \xi}^{\eta}}=\gamma_{\eta \beta}^{k} \tilde{r}_{k}^{\alpha \xi \beta}-\gamma_{k \beta}^{\alpha} \tilde{r}_{\eta}{ }^{k \xi \beta} .
$$

On the other hand, the covariant divergence of a tensor density $\tilde{r}_{\eta}{ }^{\alpha \xi \mu}$ is given by

$$
\begin{aligned}
\left(\tilde{r}_{\eta}^{\alpha \xi \beta}\right)_{; \beta}= & \left(\tilde{r}_{\eta}^{\alpha \xi \beta}\right)_{, \beta}-\gamma_{\eta \beta}^{k} \tilde{r}_{k}^{\alpha \xi \beta}+\gamma_{\beta k}^{\alpha} \tilde{r}_{\eta}^{k \xi \beta} \\
& +\gamma_{\beta k}^{\xi} \tilde{r}_{\eta}^{\alpha k \beta}+\underline{\gamma}_{\beta k^{\beta} \eta}^{\alpha \xi k}-\underline{\gamma}_{k \beta}^{k} \tilde{r}_{\eta}^{\alpha \xi \beta} .
\end{aligned}
$$

The last two terms cancel as usual for the divergence of a tensor density. The field equation (31) is thus equivalently expressed in terms of the covariant derivative as

$$
\begin{aligned}
\left(\tilde{r}_{\eta}{ }^{\alpha \xi \beta}\right)_{; \beta} & =\gamma_{k \beta}^{\alpha} \tilde{r}_{\eta}^{k \beta \xi}+\gamma_{\beta k}^{\alpha} \tilde{r}_{\eta}^{k \xi \beta}+\gamma_{\beta k}^{\xi} \tilde{r}_{\eta}^{\alpha k \beta} \\
& =\left(\gamma_{k \beta}^{\alpha}-\gamma_{\beta k}^{\alpha}\right) \tilde{r}_{\eta}^{k \beta \xi}-\gamma_{k \beta}^{\xi} \tilde{r}_{\eta}{ }^{\alpha k \beta} .
\end{aligned}
$$

As $\tilde{r}_{\eta}{ }^{\alpha k \beta}$ is skew-symmetric in $k, \beta$ according to the first canonical equation (30), the contraction with $\gamma_{k \beta}^{\xi}$ in the rightmost term of Eq. (32) extracts the skew-symmetric part of the connection coefficients, hence the torsion tensor $s^{\xi}{ }_{k \beta}=$ $\frac{1}{2}\left(\gamma_{k \beta}^{\xi}-\gamma_{\beta k}^{\xi}\right)$. Thus, Eq. (32) is actually a tensor equation $\left(\tilde{r}_{\eta}^{\alpha \xi \beta}\right)_{; \beta}$

$= \begin{cases}2 s^{\alpha}{ }_{k \beta} \tilde{r}_{\eta}{ }^{k \beta \xi}-s_{k \beta}^{\xi} \tilde{r}_{\eta}{ }^{\alpha k \beta} & \text { in general } \\ 0 & \text { for a torsion-free space-time. }\end{cases}$

The canonical equation for the space-time coefficients follows as

$$
\frac{\partial x^{\nu}}{\partial y^{\mu}}=-\frac{\partial \mathcal{H}_{\mathrm{e}}}{\partial \tilde{t}_{\nu}{ }^{\mu}}=\frac{\partial x^{\nu}}{\partial y^{\mu}} .
$$

As a common feature of all trivial extended Hamiltonians, no substantial equation for the space-time coefficients emerges but only an identity that allows for arbitrary space-time dynamics.
As $\mathcal{H}_{\mathrm{e}}$ does not depend on the $x^{\nu}$, the canonical equation for the $\tilde{t}_{\nu}{ }^{\mu}$ follows as

$$
\frac{\partial \tilde{t}_{\nu}^{\alpha}}{\partial y^{\alpha}}=-\frac{\partial \mathcal{H}_{\mathrm{e}}}{\partial x^{\nu}}=0
$$

The explicit representation of $\tilde{t}_{\nu}{ }^{\alpha}$ will be derived in Eq. (37) from the Lagrangian $\mathcal{L}_{\mathrm{e}}$ that follows from $\mathcal{H}_{\mathrm{e}}$ by means of the Legendre transformation prescription (14).

\section{FORM-INVARIANT LAGRANGIAN, EULER-LAGRANGE EQUATIONS}

The extended Lagrangian $\mathcal{L}_{\mathrm{e}}$ corresponding to the forminvariant Hamiltonian from (29) is obtained by means of the regular Legendre transformation

$$
\begin{aligned}
\mathcal{L}_{\mathrm{e}}= & \tilde{r}_{\eta}{ }^{\alpha \xi \mu} \frac{\partial \gamma^{\eta}{ }_{\alpha \xi}}{\partial x^{\mu}}-\tilde{t}_{\alpha}{ }^{\beta} \frac{\partial x^{\alpha}}{\partial y^{\beta}}-\mathcal{H}_{\mathrm{e}} \\
= & \frac{1}{4} \tilde{r}_{\eta}{ }^{\alpha \xi \mu} r^{\eta}{ }_{\alpha \xi \mu}-\frac{1}{2} \tilde{r}_{\eta}{ }^{\alpha \xi \mu} \\
& \times\left(\frac{\partial \gamma^{\eta}{ }_{\alpha \mu}}{\partial x^{\xi}}-\frac{\partial \gamma_{\alpha \xi}^{\eta}}{\partial x^{\mu}}+\gamma_{\alpha \mu}^{k} \gamma_{k \xi}^{\eta}-\gamma_{\alpha \xi}^{k} \gamma_{k \mu}^{\eta}\right) \\
= & -\frac{1}{4} \tilde{r}_{\eta}{ }^{\alpha \xi \mu} r_{\alpha \xi \mu}^{\eta} .
\end{aligned}
$$

Note that-in contrast to the Hamiltonian description - the curvature tensor $r_{\alpha \xi \mu}^{\eta}$ does not constitute a proper dynamical variable but only abbreviates the particular combination (30) of the connection coefficients and their respective derivatives-which comprise in conjunction with the spacetime coefficients the actual dynamical variables of the Lagrangian description. The dependence of $\mathcal{L}_{\mathrm{e}}$ on the space-time coefficients is expressed implicitly in terms of metric tensors

$$
\begin{gathered}
\mathcal{L}_{\mathrm{e}}\left(\gamma_{\alpha \xi}^{\eta}, \frac{\partial \gamma_{\alpha \xi}^{\eta}}{\partial x^{\nu}}, \frac{\partial x^{\mu}}{\partial y^{\nu}}\right)=-\frac{1}{4} r_{\eta}{ }^{\alpha \xi \mu} r_{\alpha \xi \mu}^{\eta} \operatorname{det} \Lambda \\
=-\frac{1}{4} g_{\kappa \eta} g^{\beta \alpha} g^{\lambda \xi} g^{\zeta \mu} r^{\kappa}{ }_{\beta \lambda \zeta} r^{\eta}{ }_{\alpha \xi \mu} \operatorname{det} \Lambda .
\end{gathered}
$$

The $\tilde{t}_{\mu}{ }^{\nu}$ represent the duals of the space-time coefficients, hence, their explicit form follows from $\mathcal{L}_{\mathrm{e}}$ according to the general rule (13). Owing to the identities (A5) and (A6), one finds for the Lagrangian (36)

$$
\begin{aligned}
-\tilde{t}_{\mu}{ }^{\nu} & =\frac{\partial \mathcal{L}_{\mathrm{e}}}{\partial\left(\frac{\partial x^{\mu}}{\partial y^{\nu}}\right)} \\
& =\left(r_{\eta}{ }^{\alpha \xi k}(x) r_{\alpha \xi \mu}^{\eta}(x)-\frac{1}{4} \delta_{\mu}^{k} r_{\eta}^{\alpha \xi \beta} r_{\alpha \xi \beta}^{\eta}\right) \frac{\partial y^{\nu}}{\partial x^{k}} \operatorname{det} \Lambda .
\end{aligned}
$$

The explicit calculation is worked out in Appendix E As $\mathcal{L}_{\mathrm{e}}$ does not depend on $x^{\mu}$, the divergence of $\tilde{t}_{\mu}{ }^{\nu}$ vanishes according to the Euler-Lagrange equation (8) and in agreement with the canonical equation (34). With Eq. (A4), its final form is then

$$
\frac{\partial}{\partial x^{k}}\left(r_{\eta}^{\alpha \xi k}(x) r_{\alpha \xi \mu}^{\eta}(x)-\frac{1}{4} \delta_{\mu}^{k} r_{\eta}^{\alpha \xi \beta} r_{\alpha \xi \beta}^{\eta}\right)=0,
$$


which has the corresponding tensor form

$$
\left(r_{\eta}^{\alpha \xi k} r_{\alpha \xi \mu}^{\eta}-\frac{1}{4} \delta_{\mu}^{k} r_{\eta}^{\alpha \xi \beta} r_{\alpha \xi \beta}^{\eta}\right)_{; k}=0 .
$$

To set up the Euler-Lagrange equation (10) for the connection coefficients, the derivative of $\mathcal{L}_{\mathrm{e}}$ from Eq. (36) with respect to the derivatives of the connection coefficients is to be calculated first

$$
\begin{aligned}
\frac{\partial \mathcal{L}_{\mathrm{e}}}{\partial\left(\frac{\partial \gamma^{k}{ }_{i j}(x)}{\partial x^{\lambda}}\right)} & =-\frac{1}{2} \tilde{r}_{\eta}{ }^{\alpha \xi \mu} \frac{\partial r^{\eta}{ }_{\alpha \xi \mu}}{\partial\left(\frac{\partial \gamma^{k}{ }_{i j}}{\partial x^{\lambda}}\right)} \\
& =-\frac{1}{2} \tilde{r}_{\eta}{ }^{\alpha \xi \mu} \delta_{k}^{\eta}\left(\delta_{\alpha}^{i} \delta_{\xi}^{\lambda} \delta_{\mu}^{j}-\delta_{\alpha}^{i} \delta_{\xi}^{j} \delta_{\mu}^{\lambda}\right) \\
& =-\frac{1}{2}\left(\tilde{r}_{k}{ }^{i \lambda j}-\tilde{r}_{k}{ }^{i j \lambda}\right) \\
& =\tilde{r}_{k}{ }^{i j \lambda}(x) .
\end{aligned}
$$

The quantities $\partial \gamma^{k}{ }_{i j}(x) / \partial x^{\lambda}$ and $\tilde{r}_{k}^{i j \lambda}(x)$ are thus dual to each other in the system described by the extended Lagrangian (36), in agreement with the corresponding canonical equation (30).

The derivative of $\mathcal{L}_{\mathrm{e}}$ with respect to the connection coefficients is

$$
\begin{aligned}
& \frac{\partial \mathcal{L}_{\mathrm{e}}}{\partial \gamma_{i j}^{k}}=-\frac{1}{2} \tilde{r}_{\eta}{ }^{\alpha \beta \mu}\left(\delta_{k}^{\xi} \delta_{\alpha}^{i} \delta_{\mu}^{j} \gamma_{\xi \beta}^{\eta}+\delta_{\xi}^{i} \delta_{\beta}^{j} \delta_{k}^{\eta} \gamma_{\alpha \mu}^{\xi}\right.\left.-\delta_{k}^{\xi} \delta_{\alpha}^{i} \delta_{\beta}^{j} \gamma_{\xi \mu}^{\eta}-\delta_{\xi}^{i} \delta_{\mu}^{j} \delta_{k}^{\eta} \gamma_{\alpha \beta}^{\xi}\right) \\
&=-\frac{1}{2}\left(\tilde{r}_{\eta}^{i \beta j} \gamma_{k \beta}^{\eta}+\tilde{r}_{k}^{\alpha j \mu} \gamma_{\alpha \mu}^{i}\right. \\
&\left.-\tilde{r}_{\eta}^{i j \mu} \gamma_{k \mu}^{\eta}-\tilde{r}_{k}^{\alpha \beta j} \gamma_{\alpha \beta}^{i}\right) \\
&=\tilde{r}_{\beta}{ }^{i j \alpha} \gamma_{k \alpha}^{\beta}+\tilde{r}_{k}^{\alpha \beta j} \gamma_{\alpha \beta}^{i} .
\end{aligned}
$$

In conjunction with Eq. (39) this yields the Euler-Lagrange equation

$$
\frac{\partial \tilde{r}_{k}^{i j \lambda}}{\partial x^{\lambda}}-\gamma_{k \alpha}^{\beta} \tilde{r}_{\beta}^{i j \alpha}-\gamma_{\alpha \beta}^{i} \tilde{r}_{k}{ }^{\alpha \beta j}=0,
$$

which actually represents a tensor equation and agrees, as expected, with the canonical equation from Eq. (31) and the subsequent field equations (33). For a torsion-free space-time, one gets in particular

$$
\left(r_{\eta}^{\alpha \xi k}\right)_{; k}=0
$$

which is a sufficient condition for Eqs. (38) to be satisfied identically [11].

The coupled set of field equations (38) and (40) must be simultaneously satisfied in order to minimize the action (9). Equation (40) provides the correlation of the connection coefficients with the metric, which here does not coincide with the usual Levi-Civita connection of standard general relativity.

\section{FORM-INVARIANT LAGRANGIAN INCLUDING MATTER}

The canonical approach to general relativity suggests that the dynamics of space-time may be described by an ex- tended Lagrangian scalar density that is quadratic in the Riemann curvature tensor. Explicitly, this "quadratic gravity" Lagrangian is proposed as

$$
\mathcal{L}_{\mathrm{e}}^{\mathrm{QG}}=\mathcal{L}_{\mathrm{e}}+\bar{\kappa} \mathcal{L}_{\mathrm{M}} \operatorname{det} \Lambda, \quad \mathcal{L}_{\mathrm{e}}=-\frac{1}{4} r_{\eta}{ }^{\alpha \xi \beta} r^{\eta}{ }_{\alpha \xi \beta} \operatorname{det} \Lambda,
$$

with $\bar{\kappa}$ a dimensionless coupling constant to the subsystem $\mathcal{L}_{\mathrm{M}}$ that describes a conventional dynamical system associated with mass and/or energy. Therefore $\mathcal{L}_{\mathrm{M}} \operatorname{det} \Lambda$ defines a trivial extended Lagrangian. Its derivative with respect to the space-time coefficients then yields the canonical energymomentum tensor $\theta_{\mu}{ }^{\nu}(x)$ of the system described by $\mathcal{L}_{\mathrm{M}}$, as derived in Eq. (A3). The derivative of $\mathcal{L}_{\mathrm{e}}^{\mathrm{QG}}$ then follows as

$$
\begin{gathered}
\frac{\partial \mathcal{L}_{\mathrm{e}}^{\mathrm{QG}}}{\partial\left(\frac{\partial x^{\mu}}{\partial y^{\nu}}\right)}=\left[r_{\eta}{ }^{\alpha \xi k}(x) r_{\alpha \xi \mu}^{\eta}(x)-\frac{1}{4} \delta_{\mu}^{k} r_{\eta}^{\alpha \xi \beta} r_{\alpha \xi \beta}^{\eta}\right. \\
\left.-\bar{\kappa} \theta_{\mu}{ }^{k}(x)\right] \frac{\partial y^{\nu}}{\partial x^{k}} \operatorname{det} \Lambda .
\end{gathered}
$$

If $\mathcal{L}_{\mathrm{M}}$ does not explicitly depend on the $x^{\mu}$, then the EulerLagrange equation for the space-time coefficients is given by

$$
\begin{gathered}
\frac{\partial}{\partial y^{\nu}}\left(\left[r_{\eta}{ }^{\alpha \xi k}(x) r_{\alpha \xi \mu}^{\eta}(x)-\frac{1}{4} \delta_{\mu}^{k} r_{\eta}{ }^{\alpha \xi \beta} r_{\alpha \xi \beta}^{\eta}\right.\right. \\
\left.\left.-\bar{\kappa} \theta_{\mu}{ }^{k}(x)\right] \frac{\partial y^{\nu}}{\partial x^{k}} \operatorname{det} \Lambda\right)=0
\end{gathered}
$$

hence by virtue of Eq. (A4) in tensor form

$$
\left(r_{\eta}^{\alpha \xi k} r_{\alpha \xi \mu}^{\eta}-\frac{1}{4} \delta_{\mu}^{k} r_{\eta}^{\alpha \xi \beta} r_{\alpha \xi \beta}^{\eta}-\bar{\kappa} \theta_{\mu}{ }^{k}\right)_{; k}=0
$$

which generalizes the field equation of the matter-free system from Eq. (38). As $\mathcal{L}_{\mathrm{M}} \operatorname{det} \Lambda$ does not depend on the connection coefficients, the corresponding Euler-Lagrange equation of $\mathcal{L}_{\mathrm{e}}^{\mathrm{QG}}$ agrees with (40). Provided that the conventional mass/energy Lagrangian $\mathcal{L}_{\mathrm{M}}$ in (41) is autonomous, hence does not explicitly depend on space-time, then both groups of covariant derivatives of (42) vanish separately

$$
\left(r_{\eta}{ }^{\alpha \xi k} r_{\alpha \xi \mu}^{\eta}-\frac{1}{4} \delta_{\mu}^{k} r_{\eta}^{\alpha \xi \beta} r_{\alpha \xi \beta}^{\eta}\right)_{; k} \equiv 0, \quad \theta_{\mu ; k}^{k} \equiv 0,
$$

which states the local conservation of energy and momentum for a closed system.

Equation (42) constitutes the equation of general relativity as derived from the extended canonical transformation of the connection coefficients. It is not assumed here that the LeviCivita connection applies for the independent geometric quantities given by the Christoffel symbols and the metric. Rather, the connection is established by Eq. (33) or by the more special equation (40) in the case of a torsion-free space-time.

\section{CONCLUSIONS}

The gauge theory that is based on the extended canonical transformation formalism suggests that general relativity is described by the Lagrangian

$$
\mathcal{L}_{\mathrm{e}}^{\mathrm{QG}}=\left(-\frac{1}{4} R_{\eta}{ }^{\alpha \xi \beta} R^{\eta}{ }_{\alpha \xi \beta}+\bar{\kappa} \mathcal{L}_{\mathrm{M}}\right) \operatorname{det} \Lambda,
$$


with $\bar{\kappa}$ a dimensionless coupling constant of the Riemann curvature tensor term and $\mathcal{L}_{\mathrm{M}}$ the conventional matter/energy Lagrangian. Following Palatini's approach, the extended canonical formalism treats the Christoffel symbols and the spacetime coefficients implicitly contained in (43) as independent quantities. This yields two independent Euler-Lagrange equations that must be simultaneously satisfied in order for the solutions to satisfy the action principle. No a priori assumptions on a correlation of the Christoffel symbols with the space-time coefficients—and hence the metric — are made.

The extended Lagrangian (43) was obtained by Legendretransforming the form-invariant extended Hamiltonian that emerged from an extended canonical transformation. The underlying extended generating function $\mathcal{F}_{2}^{\mu}$ was set up to yield the required transformation rule for the connection coefficients. The latter act as "gauge coefficients" that render globally (Lorentz-)invariant systems form-invariant under the corresponding local transformation group, i.e. the diffeomorphism group. The form-invariant extended Hamiltonian could then be deduced from the general rules for extended canonical transformations in the realm of field theory.

As was noted by C. Lanczos [12], the Lagrangian of general relativity should be a quadratic function of the curvature tensor elements in order to obey the principle of scaleindependence, which means that the value of the action integral (96) "should not depend on the arbitrary units employed in measuring the lengths of the space-time manifold." The Lagrangian (43) derived here meets this requirement. Summarizing, the theory has the following properties

1. The space-time geometry is of Riemannian type, with a torsion of space-time not excluded a priori. Hence, the theory does not presuppose nor require the equivalence principle-i.e. the equivalence of gravitational and inertial mass-to hold strictly.

2. The theory is based on the action principle, which yields field equations for the space-time and the connection coefficients and hence the metric tensor.

3. The general principle of relativity is satisfied, hence, the theory is form-invariant under the canonical transformation of the connection coefficients and their conjugates-which ensures the action principle to be maintained. This can be regarded as the realization of the gauge principle for a diffeomorphism-invariant theory, with the connection coefficients playing the role of "gauge fields."

4. The principle of scale invariance holds, hence the theory is form-invariant under transformations of the length scales of the space-time manifold.

5. The theory is unambiguous in the sense that no other functions of the Riemann curvature tensor emerge from the canonical transformation formalism.

6. In contrast to standard general relativity that is based on the postulated Einstein-Hilbert action, the Lagrangian (36) is derived, based on the requirement of its form-invariance under the canonical transformation of the connection coefficients, and hence on the general principle of relativity. Moreover, no ad hoc assumption concerning the relation of the connection coefficients with the metric is incorporated into the formalism. Instead, it is the canonical equation (33) that provides the correlation of the connection coefficients with the metric.

7. For the source-free case $\left(\mathcal{L}_{\mathrm{M}} \equiv 0\right)$, the theory is compatible with standard general relativity in the torsionfree limit as it possesses then the Schwarzschild metric as a solution [11]. For $\mathcal{L}_{\mathrm{M}} \neq 0$, the solution is expected to differ from that of standard general relativity - especially if a torsion of space-time is not to be excluded a priori.

8. A quantized theory that is based on the Lagrangian (43) is renormalizable [13]. It is a well-known fact that the energy of a gravitational field is not localizable, hence that the energy-momentum (pseudo-)tensor of the gravitational field depends on the frame of reference. In a quantized theory of gravity, this would mean that the hypothetical interaction bosons (the "gravitons") are the quanta of a nontensorial classical "field" that is represented by the connection coefficients $\Gamma_{\mu \nu}^{\xi}$.

Remarkably, a Lagrangian of the form of Eq. (41) that is quadratic in the curvature tensor was already proposed by A. Einstein in a personal letter to Hermann Weyl, dated March 08, 1918 [14], reasoning analogies with other classical field theories.

\section{Appendix A: USEFUL IDENTITIES}

In order to show that the conventional Lagrangian $\mathcal{L}$ description of a dynamical system is compatible with the corresponding description in terms of extended Lagrangians, we must make use of the following identities

$$
\begin{aligned}
\frac{\partial \phi_{I}}{\partial x^{\alpha}}= & \frac{\partial \phi_{I}}{\partial y^{i}} \frac{\partial y^{i}}{\partial x^{\alpha}} \\
& \Rightarrow \frac{\partial\left(\frac{\partial \phi_{I}}{\partial x^{\alpha}}\right)}{\partial\left(\frac{\partial \phi_{I}}{\partial y^{\nu}}\right)}=\delta_{i}^{\nu} \frac{\partial y^{i}}{\partial x^{\alpha}}=\frac{\partial y^{\nu}}{\partial x^{\alpha}} \\
& \Rightarrow \frac{\partial\left(\frac{\partial \phi_{I}}{\partial x^{\alpha}}\right)}{\partial\left(\frac{\partial y^{\nu}}{\partial x^{\mu}}\right)}=\frac{\partial \phi_{I}}{\partial y^{i}} \delta_{\nu}^{i} \delta_{\alpha}^{\mu}=\frac{\partial \phi_{I}}{\partial y^{\nu}} \delta_{\alpha}^{\mu} \\
\frac{\partial x^{\nu}}{\partial y^{k}} \frac{\partial y^{k}}{\partial x^{\mu}}= & \delta_{\mu}^{\nu} \\
& \Rightarrow \frac{\partial\left(\frac{\partial y^{\nu}}{\partial x^{\mu}}\right)}{\partial\left(\frac{\partial x^{\alpha}}{\partial y^{\beta}}\right)}=-\frac{\partial y^{\nu}}{\partial x^{\alpha}} \frac{\partial y^{\beta}}{\partial x^{\mu}} \\
& \Rightarrow \frac{\partial^{2} y^{k}}{\partial x^{\mu} \partial x^{\xi}} \frac{\partial x^{\nu}}{\partial y^{k}}=-\frac{\partial^{2} x^{\nu}}{\partial y^{j} \partial y^{k}} \frac{\partial y^{j}}{\partial x^{\xi}} \frac{\partial y^{k}}{\partial x^{\mu}}
\end{aligned}
$$




$$
\begin{aligned}
\frac{\partial\left(\frac{\partial \phi_{I}}{\partial x^{\alpha}}\right)}{\partial\left(\frac{\partial x^{\mu}}{\partial y^{\nu}}\right)} & =\frac{\partial\left(\frac{\partial \phi_{I}}{\partial x^{\alpha}}\right)}{\partial\left(\frac{\partial y^{j}}{\partial x^{i}}\right)} \frac{\partial\left(\frac{\partial y^{j}}{\partial x^{2}}\right)}{\partial\left(\frac{\partial x^{\mu}}{\partial y^{\nu}}\right)} \\
& =-\frac{\partial \phi_{I}}{\partial y^{j}} \frac{\partial y^{j}}{\partial x^{\mu}} \frac{\partial y^{\nu}}{\partial x^{\alpha}}=-\frac{\partial \phi_{I}}{\partial x^{\mu}} \frac{\partial y^{\nu}}{\partial x^{\alpha}} .
\end{aligned}
$$

Frequently, the derivative of the Jacobi determinant with respect to the space-time coefficients needs to be inserted. This quantity is easiest calculated on the basis of the general formula for a determinant of an $n \times n$ matrix $A=\left(a_{i k}\right)$. With $S_{n}$ the set of all permutations $\pi$ of the numbers $1, \ldots, n$, the determinant is given by the sum over all $\pi \in S_{n}$

$$
\operatorname{det} A=\sum_{\pi \in S_{n}} \operatorname{sgn} \pi a_{1 \pi(1)} \ldots a_{n \pi(n)} .
$$

Herein, $\operatorname{sgn} \pi= \pm 1$ depending on the permutation to consist of an even or odd number of elementary transpositions of pairs of numbers. In the first case, $\operatorname{sgn} \pi=+1$ whereas $\operatorname{sgn} \pi=$ -1 in the latter. This means for $\operatorname{det} \Lambda$

$$
\operatorname{det} \Lambda=\sum_{\pi \in S_{n+1}} \operatorname{sgn} \pi \frac{\partial x^{0}}{\partial y^{\pi(0)}} \cdots \frac{\partial x^{n}}{\partial y^{\pi(n)}} .
$$

With the sum over all $\alpha$, the expression

$$
\begin{aligned}
& \frac{\partial \operatorname{det} \Lambda}{\partial\left(\frac{\partial x^{\mu}}{\partial y^{\alpha}}\right)} \frac{\partial x^{\nu}}{\partial y^{\alpha}} \\
= & \left(\sum_{\pi \in S_{n+1}} \operatorname{sgn} \pi \frac{\partial x^{0}}{\partial y^{\pi(0)}} \cdots \frac{\partial x^{\mu-1}}{\partial y^{\pi(\mu-1)}} \delta_{\pi(\mu)}^{\alpha}\right. \\
& \left.\times \frac{\partial x^{\mu+1}}{\partial y^{\pi(\mu+1)}} \cdots \frac{\partial x^{n}}{\partial y^{\pi(n)}}\right) \frac{\partial x^{\nu}}{\partial y^{\alpha}} \\
= & \sum_{\pi \in S_{n+1}} \operatorname{sgn} \pi \frac{\partial x^{0}}{\partial y^{\pi(0)}} \cdots \frac{\partial x^{\mu-1}}{\partial y^{\pi(\mu-1)}} \frac{\partial x^{\nu}}{\partial y^{\pi(\mu)}} \frac{\partial x^{\mu+1}}{\partial y^{\pi(\mu+1)}} \cdots \frac{\partial x^{n}}{\partial y^{\pi(n)}}
\end{aligned}
$$

is either zero for $\nu \neq \mu$ as the derivative of $x^{\nu}$ then occurs twice or equal to $\operatorname{det} \Lambda$ for $\nu=\mu$ as the derivative of $x^{\mu}$ is recovered and thus yields the initial expression for the determinant $\operatorname{det} \Lambda$. Thus

$$
\frac{\partial \operatorname{det} \Lambda}{\partial\left(\frac{\partial x^{\mu}}{\partial y^{\alpha}}\right)} \frac{\partial x^{\nu}}{\partial y^{\alpha}}=\delta_{\mu}^{\nu} \operatorname{det} \Lambda,
$$

hence

$$
\frac{\partial \operatorname{det} \Lambda}{\partial\left(\frac{\partial x^{\mu}}{\partial y^{\nu}}\right)}=\frac{\partial y^{\nu}}{\partial x^{\mu}} \operatorname{det} \Lambda .
$$

The correlation (6) of the trivial extended Lagrangian $\mathcal{L}_{\mathrm{e}}$ and conventional Lagrangian $\mathcal{L}$ emerges from the requirement of Eq. (4) to yield the identical action $S$, hence to describe the same physical system. The derivative of a trivial extended Lagrangian with respect to the space-time coefficients yields the canonical energy-momentum tensor. Explicitly,

$$
\begin{aligned}
\frac{\partial \mathcal{L}_{\mathrm{e}}^{\text {triv }}}{\partial\left(\frac{\partial x^{\mu}}{\partial y^{\nu}}\right)} & =\mathcal{L} \frac{\partial \operatorname{det} \Lambda}{\partial\left(\frac{\partial x^{\mu}}{\partial y^{\nu}}\right)}+\frac{\partial \mathcal{L}}{\partial\left(\frac{\partial \phi_{I}}{\partial x^{\alpha}}\right)} \frac{\partial\left(\frac{\partial \phi_{I}}{\partial x^{\alpha}}\right)}{\partial\left(\frac{\partial x^{\mu}}{\partial y^{\nu}}\right)} \operatorname{det} \Lambda \\
& =\mathcal{L} \frac{\partial y^{\nu}}{\partial x^{\mu}} \operatorname{det} \Lambda-\frac{\partial \mathcal{L}}{\partial\left(\frac{\partial \phi_{I}}{\partial x^{\alpha}}\right)} \frac{\partial \phi_{I}}{\partial x^{\mu}} \frac{\partial y^{\nu}}{\partial x^{\alpha}} \operatorname{det} \Lambda \\
& =\left(\delta_{\mu}^{i} \mathcal{L}-\frac{\partial \mathcal{L}}{\partial\left(\frac{\partial \phi_{I}}{\partial x^{\alpha}}\right)} \frac{\partial \phi_{I}}{\partial x^{\mu}}\right) \frac{\partial y^{\nu}}{\partial x^{\alpha}} \operatorname{det} \Lambda \\
& =-\theta_{\mu}{ }^{\alpha}(x) \frac{\partial y^{\nu}}{\partial x^{\alpha}} \operatorname{det} \Lambda=-\tilde{\theta}_{\mu}{ }^{\alpha}(x) \frac{\partial y^{\nu}}{\partial x^{\alpha}},
\end{aligned}
$$

where $\theta_{\mu}{ }^{\nu}$ denotes the energy-momentum tensor, and $\tilde{\theta}_{\mu}{ }^{\nu}$ the corresponding tensor density at the same space-time location.

A frequently used identity follows as

$$
\begin{aligned}
& \frac{\partial}{\partial y^{\alpha}} \frac{\partial \operatorname{det} \Lambda}{\partial\left(\frac{\partial x^{\mu}}{\partial y^{\alpha}}\right)}=\frac{\partial}{\partial y^{\alpha}}\left(\frac{\partial y^{\alpha}}{\partial x^{\mu}} \operatorname{det} \Lambda\right) \\
& =\frac{\partial^{2} y^{\alpha}}{\partial x^{\mu} \partial x^{\beta}} \frac{\partial x^{\beta}}{\partial y^{\alpha}} \operatorname{det} \Lambda+\frac{\partial y^{\alpha}}{\partial x^{\mu}} \frac{\partial \operatorname{det} \Lambda}{\partial\left(\frac{\partial x^{i}}{\partial y^{j}}\right)} \frac{\partial^{2} x^{i}}{\partial y^{j} \partial y^{\alpha}} \\
& =(\frac{\partial^{2} y^{\alpha}}{\partial x^{\mu} \partial x^{\beta}} \frac{\partial x^{\beta}}{\partial y^{\alpha}}+\underbrace{\frac{\partial y^{\alpha}}{\partial x^{\mu}} \frac{\partial y^{j}}{\partial x^{i}} \frac{\partial^{2} x^{i}}{\partial y^{j} \partial y^{\alpha}}}_{\underline{\underline{\underline{A}}}-\frac{\partial^{2} y^{\alpha}}{\partial x^{\mu} \partial x^{\beta}} \frac{\partial x^{\beta}}{\partial y^{\alpha}}}) \operatorname{det} \Lambda \\
& \equiv 0 \text {. }
\end{aligned}
$$

This identity is used for setting up the extended set of EulerLagrange equations.

The derivative of the contravariant metric with respect to the space-time coefficients follows as

$$
\begin{aligned}
& \frac{\partial g^{\mu \nu}(y)}{\partial\left(\frac{\partial x^{\alpha}}{\partial y^{\beta}}\right)}=g^{i j}(x)\left[\frac{\partial\left(\frac{\partial y^{\mu}}{\partial x^{i}}\right)}{\partial\left(\frac{\partial x^{\alpha}}{\partial y^{\beta}}\right)} \frac{\partial y^{\nu}}{\partial x^{j}}+\frac{\partial y^{\mu}}{\partial x^{i}} \frac{\partial\left(\frac{\partial y^{\nu}}{\partial x^{j}}\right)}{\partial\left(\frac{\partial x^{\alpha}}{\partial y^{\beta}}\right)}\right] \\
= & -g^{a b}(y) \frac{\partial x^{i}}{\partial y^{a}} \frac{\partial x^{j}}{\partial y^{b}}\left(\frac{\partial y^{\mu}}{\partial x^{\alpha}} \frac{\partial y^{\beta}}{\partial x^{i}} \frac{\partial y^{\nu}}{\partial x^{j}}+\frac{\partial y^{\mu}}{\partial x^{i}} \frac{\partial y^{\nu}}{\partial x^{\alpha}} \frac{\partial y^{\beta}}{\partial x^{j}}\right) \\
= & -g^{\beta \nu}(y) \frac{\partial y^{\mu}}{\partial x^{\alpha}}-g^{\mu \beta}(y) \frac{\partial y^{\nu}}{\partial x^{\alpha}} \\
= & -\left(\delta_{j}^{\mu} g^{\beta \nu}(y)+\delta_{j}^{\nu} g^{\mu \beta}(y)\right) \frac{\partial y^{j}}{\partial x^{\alpha}} .
\end{aligned}
$$

The derivative of the covariant metric with respect to the space-time coefficients is then

$$
\begin{aligned}
\frac{\partial g_{\mu \nu}(y)}{\partial\left(\frac{\partial x^{\alpha}}{\partial y^{\beta}}\right)} & =g_{i j}(x)\left[\frac{\partial\left(\frac{\partial x^{i}}{\partial y^{\mu}}\right)}{\partial\left(\frac{\partial x^{\alpha}}{\partial y^{\beta}}\right)} \frac{\partial x^{j}}{\partial y^{\nu}}+\frac{\partial x^{i}}{\partial y^{\mu}} \frac{\partial\left(\frac{\partial x^{j}}{\partial y^{\nu}}\right)}{\partial\left(\frac{\partial x^{\alpha}}{\partial y^{\beta}}\right)}\right] \\
& =g_{a b}(y) \frac{\partial y^{a}}{\partial x^{i}} \frac{\partial y^{b}}{\partial x^{j}}\left(\delta_{\alpha}^{i} \delta_{\mu}^{\beta} \frac{\partial x^{j}}{\partial y^{\nu}}+\frac{\partial x^{i}}{\partial y^{\mu}} \delta_{\alpha}^{j} \delta_{\nu}^{\beta}\right) \\
& =\left(\delta_{\mu}^{\beta} g_{j \nu}(y)+\delta_{\nu}^{\beta} g_{\mu j}(y)\right) \frac{\partial y^{j}}{\partial x^{\alpha}} .
\end{aligned}
$$




\section{Appendix B: CONNECTION COEFFICIENTS}

The derivatives of a vector $a_{\mu}$ do not transform as tensors

$$
\frac{\partial a_{\mu}(X)}{\partial X^{\nu}}=\frac{\partial a_{i}(x)}{\partial x^{j}} \frac{\partial x^{j}}{\partial X^{\nu}} \frac{\partial x^{i}}{\partial X^{\mu}}+a_{i}(x) \frac{\partial^{2} x^{i}}{\partial X^{\mu} \partial X^{\nu}},
$$

provided that the reference system $x(y)$ is curved with respect to the reference system $X(y)$, which means that not all second derivatives of the $x^{\mu}$ in (B1) vanish.

Equation (B1) can be converted into a tensor equation by introducing connection coefficients $\gamma_{\alpha \beta}^{\mu}(x)$ and $\Gamma_{\alpha \beta}^{\mu}(X)$

$$
\begin{aligned}
\frac{\partial a_{\mu}(X)}{\partial X^{\nu}}= & \frac{\partial x^{j}}{\partial X^{\nu}} \frac{\partial x^{i}}{\partial X^{\mu}} \frac{\partial a_{i}(x)}{\partial x^{j}}+a_{k}(x) \frac{\partial^{2} x^{k}}{\partial X^{\mu} \partial X^{\nu}} \\
= & \frac{\partial x^{j}}{\partial X^{\nu}} \frac{\partial x^{i}}{\partial X^{\mu}}\left(\frac{\partial a_{i}(x)}{\partial x^{j}}-a_{k}(x) \gamma_{i j}^{k}(x)\right) \\
& +a_{k}(X) \Gamma_{\mu \nu}^{k}(X) .
\end{aligned}
$$

Then

$$
\begin{aligned}
& \frac{\partial a_{\mu}(X)}{\partial X^{\nu}}-a_{k}(X) \Gamma_{\mu \nu}^{k}(X) \\
& \quad=\frac{\partial x^{j}}{\partial X^{\nu}} \frac{\partial x^{i}}{\partial X^{\mu}}\left(\frac{\partial a_{i}(x)}{\partial x^{j}}-a_{k}(x) \gamma_{i j}^{k}(x)\right)
\end{aligned}
$$

which shows that the quantity

$$
a_{i ; j} \equiv \frac{\partial a_{i}(x)}{\partial x^{j}}-a_{k}(x) \gamma_{i j}^{k}(x)
$$

transforms as a tensor, provided that the connection coefficients transform as

$$
\begin{aligned}
a_{k}(x) \frac{\partial^{2} x^{k}}{\partial X^{\mu} \partial X^{\nu}}= & a_{k}(x) \frac{\partial x^{k}}{\partial X^{j}} \Gamma_{\mu \nu}^{j}(X) \\
& -\frac{\partial x^{j}}{\partial X^{\nu}} \frac{\partial x^{i}}{\partial X^{\mu}} a_{k}(x) \gamma_{i j}^{k}(x) .
\end{aligned}
$$

As this equation holds for arbitrary $a_{k}(x)$, it follows that

$$
\Gamma_{\mu \nu}^{j}(X) \frac{\partial x^{k}}{\partial X^{j}}=\gamma_{i j}^{k}(x) \frac{\partial x^{i}}{\partial X^{\mu}} \frac{\partial x^{j}}{\partial X^{\nu}}+\frac{\partial^{2} x^{k}}{\partial X^{\mu} \partial X^{\nu}},
$$

and finally after contraction with $\partial X^{\alpha} / \partial x^{k}$,

$$
\Gamma_{\mu \nu}^{\alpha}(X)=\gamma_{i j}^{k}(x) \frac{\partial x^{i}}{\partial X^{\mu}} \frac{\partial x^{j}}{\partial X^{\nu}} \frac{\partial X^{\alpha}}{\partial x^{k}}+\frac{\partial^{2} x^{k}}{\partial X^{\mu} \partial X^{\nu}} \frac{\partial X^{\alpha}}{\partial x^{k}} .
$$

This equation provides the unique correlation of the spacetime coefficients and their derivatives with the connection coefficients. The connection coefficients are symmetric in their lower indices for torsion-free space. Otherwise, their skewsymmetric part define the torsion tensor.

\section{Appendix C: EULER-LAGRANGE EQUATIONS FOR SPACE-TIME COEFFICIENTS AND CONNECTION COEFFICIENTS}

Given an extended Lagrangian that depends on the spacetime event, the connection coefficients, and their respective space-time derivatives. The action functional over a spacetime region $R$ is then

$$
S=\int_{R} \mathcal{L}_{\mathrm{e}}\left(\gamma_{\alpha \xi}^{\eta}(y), \frac{\partial \gamma_{\alpha \xi}^{\eta}}{\partial y^{\nu}}, x^{\mu}(y), \frac{\partial x^{\mu}}{\partial y^{\nu}}\right) \mathrm{d}^{4} y, \quad \delta S \stackrel{!}{=} 0 .
$$

The variation of $\mathcal{L}_{\mathrm{e}}$ follows as

$$
\begin{aligned}
\delta \mathcal{L}_{\mathrm{e}}=\frac{\partial \mathcal{L}_{\mathrm{e}}}{\partial x^{\alpha}} \delta x^{\alpha} & +\frac{\partial \mathcal{L}_{\mathrm{e}}}{\partial\left(\frac{\partial x^{\alpha}}{\partial y^{\beta}}\right)} \delta\left(\frac{\partial x^{\alpha}}{\partial y^{\beta}}\right)+\frac{\partial \mathcal{L}_{\mathrm{e}}}{\partial \gamma^{\eta}{ }_{\alpha \xi}} \delta \gamma^{\eta}{ }_{\alpha \xi} \\
& +\frac{\partial \mathcal{L}_{\mathrm{e}}}{\partial\left(\frac{\partial \gamma^{\eta}{ }_{\alpha \xi}}{\partial y^{\beta}}\right)} \delta\left(\frac{\partial \gamma^{\eta}{ }_{\alpha \xi}}{\partial y^{\beta}}\right) .
\end{aligned}
$$

As the independent variables $y^{\mu}$ are not varied, the differentiation with respect to $y^{\beta}$ may be interchanged with the variation. This yields the equivalent representations of $\delta \mathcal{L}_{\mathrm{e}}$

$$
\begin{aligned}
\delta \mathcal{L}_{\mathrm{e}}=\frac{\partial \mathcal{L}_{\mathrm{e}}}{\partial x^{\alpha}} \delta x^{\alpha} & +\frac{\partial \mathcal{L}_{\mathrm{e}}}{\partial\left(\frac{\partial x^{\alpha}}{\partial y^{\beta}}\right)} \frac{\partial\left(\delta x^{\alpha}\right)}{\partial y^{\beta}}+\frac{\partial \mathcal{L}_{\mathrm{e}}}{\partial \gamma^{\eta}{ }_{\alpha \xi}} \delta \gamma^{\eta}{ }_{\alpha \xi} \\
& +\frac{\partial \mathcal{L}_{\mathrm{e}}}{\partial\left(\frac{\partial \gamma^{\eta}{ }_{\alpha \xi}}{\partial y^{\beta}}\right)} \frac{\partial\left(\delta \gamma^{\eta}{ }_{\alpha \xi}\right)}{\partial y^{\beta}}
\end{aligned}
$$

and

$$
\begin{aligned}
\delta \mathcal{L}_{\mathrm{e}}= & \left(\frac{\partial \mathcal{L}_{\mathrm{e}}}{\partial x^{\alpha}}-\frac{\partial}{\partial y^{\beta}} \frac{\partial \mathcal{L}_{\mathrm{e}}}{\partial\left(\frac{\partial x^{\alpha}}{\partial y^{\beta}}\right)}\right) \delta x^{\alpha}+\frac{\partial}{\partial y^{\beta}}\left(\frac{\partial \mathcal{L}_{\mathrm{e}}}{\partial\left(\frac{\partial x^{\alpha}}{\partial y^{\beta}}\right)} \delta x^{\alpha}\right) \\
& +\left(\frac{\partial \mathcal{L}_{\mathrm{e}}}{\partial \gamma^{\eta}{ }_{\alpha \xi}}-\frac{\partial}{\partial y^{\beta}} \frac{\partial \mathcal{L}_{\mathrm{e}}}{\partial\left(\frac{\partial \gamma^{\eta}{ }_{\alpha \xi}}{\partial y^{\beta}}\right)}\right) \delta \gamma^{\eta}{ }_{\alpha \xi} \\
& +\frac{\partial}{\partial y^{\beta}}\left(\frac{\partial \mathcal{L}_{\mathrm{e}}}{\partial\left(\frac{\partial \gamma^{\eta}{ }_{\alpha \xi}}{\partial y^{\beta}}\right)} \delta \gamma^{\eta}{ }_{\alpha \xi}\right) .
\end{aligned}
$$

According to Gauss' theorem, the divergence terms can be converted into surface terms in the action functional. This means explicitly

$$
\begin{aligned}
& \int_{R} \frac{\partial}{\partial y^{\beta}}\left(\frac{\partial \mathcal{L}_{\mathrm{e}}}{\partial\left(\frac{\partial x^{\alpha}}{\partial y^{\beta}}\right)} \delta x^{\alpha}\right) \mathrm{d}^{4} y=\oint_{\partial R} \frac{\partial \mathcal{L}_{\mathrm{e}}}{\partial\left(\frac{\partial x^{\alpha}}{\partial y^{\beta}}\right)} \delta x^{\alpha} \mathrm{d} S_{\beta} \\
& \int_{R} \frac{\partial}{\partial y^{\beta}}\left(\frac{\partial \mathcal{L}_{\mathrm{e}}}{\partial\left(\frac{\partial \gamma^{\eta}{ }_{\alpha \xi}}{\partial y^{\beta}}\right)} \delta \gamma^{\eta}{ }_{\alpha \xi}\right) \mathrm{d}^{4} y=\oint_{\partial R} \frac{\partial \mathcal{L}_{\mathrm{e}}}{\partial\left(\frac{\partial \gamma^{\eta}{ }_{\alpha \xi}}{\partial y^{\beta}}\right)} \delta \gamma^{\eta}{ }_{\alpha \xi} \mathrm{d} S_{\beta}
\end{aligned}
$$

with $\mathrm{d} S_{\beta}$ denoting the $\beta$ component of the normal vector on the boundary surface $\partial R$ of the volume $R$. Both surface integrals vanish since on the boundary of the space-time region $R$

$$
\left.\delta x^{\alpha}\right|_{\partial R}=0,\left.\quad \delta \gamma^{\eta}{ }_{\alpha \xi}\right|_{\partial R}=0
$$


which implies that the space-time geometry is flat on $\partial R$. The action principle $\delta S \stackrel{!}{=} 0$ then reduces to

$$
\begin{aligned}
0 \stackrel{!}{=} \int_{R}\left[\left(\frac{\partial \mathcal{L}_{\mathrm{e}}}{\partial x^{\alpha}}-\frac{\partial}{\partial y^{\beta}} \frac{\partial \mathcal{L}_{\mathrm{e}}}{\partial\left(\frac{\partial x^{\alpha}}{\partial y^{\beta}}\right)}\right) \delta x^{\alpha}\right. \\
\left.\quad+\left(\frac{\partial \mathcal{L}_{\mathrm{e}}}{\partial \gamma^{\eta}{ }_{\alpha \xi}}-\frac{\partial}{\partial y^{\beta}} \frac{\partial \mathcal{L}_{\mathrm{e}}}{\partial\left(\frac{\partial \gamma^{\eta}{ }_{\alpha \xi}}{\partial y^{\beta}}\right)}\right) \delta \gamma^{\eta}{ }_{\alpha \xi}\right] \mathrm{d}^{4} y
\end{aligned}
$$

As the variations $\delta x^{\alpha}$ and $\delta \gamma^{\eta}{ }_{\alpha \xi}$ are arbitrary and mutually independent by assumption, this condition can only be satisfied if the expressions in parentheses vanish simultaneously

$$
\frac{\partial}{\partial y^{\beta}} \frac{\partial \mathcal{L}_{\mathrm{e}}}{\partial\left(\frac{\partial x^{\alpha}}{\partial y^{\beta}}\right)}-\frac{\partial \mathcal{L}_{\mathrm{e}}}{\partial x^{\alpha}}=0, \quad \frac{\partial}{\partial y^{\beta}} \frac{\partial \mathcal{L}_{\mathrm{e}}}{\partial\left(\frac{\partial \gamma^{\eta}{ }^{\beta}}{\partial y^{\beta}}\right)}-\frac{\partial \mathcal{L}_{\mathrm{e}}}{\partial \gamma^{\eta}{ }_{\alpha \xi}}=0 .
$$

These equations are the Euler-Lagrange equations for the space-time and the connection coefficients.

\section{Appendix D: EXPLICIT CALCULATION OF THE TRANSFORMATION RULE 27)}

In expanded form, Eq. (26) reads

$$
\begin{aligned}
\tilde{T}_{\alpha}{ }^{\beta} \frac{\partial X^{\alpha}}{\partial y^{\beta}}-\tilde{t}_{\alpha}{ }^{\beta} \frac{\partial x^{\alpha}}{\partial y^{\beta}}=\tilde{R}_{\eta}{ }^{\alpha \xi \mu}\left[\gamma^{k}{ }_{i j}(\right. & \left.\frac{\partial^{2} X^{\eta}}{\partial x^{k} \partial x^{\nu}} \frac{\partial x^{\nu}}{\partial X^{\mu}} \frac{\partial x^{i}}{\partial X^{\alpha}} \frac{\partial x^{j}}{\partial X^{\xi}}+\frac{\partial^{2} x^{i}}{\partial X^{\alpha} \partial X^{\mu}} \frac{\partial X^{\eta}}{\partial x^{k}} \frac{\partial x^{j}}{\partial X^{\xi}}+\frac{\partial^{2} x^{j}}{\partial X^{\xi} \partial X^{\mu}} \frac{\partial X^{\eta}}{\partial x^{k}} \frac{\partial x^{i}}{\partial X^{\alpha}}\right) \\
& \left.+\frac{\partial^{2} X^{\eta}}{\partial x^{k} \partial x^{\nu}} \frac{\partial^{2} x^{k}}{\partial X^{\alpha} \partial X^{\xi}} \frac{\partial x^{\nu}}{\partial X^{\mu}}+\frac{\partial^{3} x^{k}}{\partial X^{\alpha} \partial X^{\xi} \partial X^{\mu}} \frac{\partial X^{\eta}}{\partial x^{k}}\right] .
\end{aligned}
$$

This expression is now split into a skew-symmetric and a symmetric part of $\tilde{R}_{\eta}{ }^{\alpha \xi \mu}$ in the indices $\xi, \mu$ according to

$$
\tilde{R}_{\eta}^{\alpha \xi \mu}=\frac{1}{2}\left(\tilde{R}_{\eta}^{\alpha \xi \mu}-\tilde{R}_{\eta}^{\alpha \mu \xi}\right)+\frac{1}{2}\left(\tilde{R}_{\eta}^{\alpha \xi \mu}+\tilde{R}_{\eta}^{\alpha \mu \xi}\right)=\tilde{R}_{\eta}{ }^{\alpha[\xi \mu]}+\tilde{R}_{\eta}{ }^{\alpha(\xi \mu)}
$$

For the skew-symmetric part, $\tilde{R}_{\eta}^{\alpha[\xi \mu]}$, the two terms in (D1) symmetric in $\xi, \mu$ vanish, hence

$$
\begin{aligned}
& \tilde{R}_{\eta}{ }^{\alpha[\xi \mu]}\left[\gamma^{k}{ }_{i j}\left(\frac{\partial^{2} X^{\eta}}{\partial x^{k} \partial x^{\nu}} \frac{\partial x^{\nu}}{\partial X^{\mu}} \frac{\partial x^{i}}{\partial X^{\alpha}} \frac{\partial x^{j}}{\partial X^{\xi}}+\frac{\partial^{2} x^{i}}{\partial X^{\alpha} \partial X^{\mu}} \frac{\partial X^{\eta}}{\partial x^{k}} \frac{\partial x^{j}}{\partial X^{\xi}}\right)+\frac{\partial^{2} X^{\eta}}{\partial x^{k} \partial x^{\nu}} \frac{\partial x^{\nu}}{\partial X^{\mu}} \frac{\partial^{2} x^{k}}{\partial X^{\alpha} \partial X^{\xi}}\right] \\
& =\tilde{R}_{\eta}{ }^{\alpha[\xi \mu]}\left[\frac{\partial^{2} X^{\eta}}{\partial x^{k} \partial x^{\nu}} \frac{\partial x^{\nu}}{\partial X^{\mu}}\left(\gamma^{k}{ }_{i j} \frac{\partial x^{i}}{\partial X^{\alpha}} \frac{\partial x^{j}}{\partial X^{\xi}}+\frac{\partial^{2} x^{k}}{\partial X^{\alpha} \partial X^{\xi}}\right)+\gamma^{k}{ }_{i j} \frac{\partial^{2} x^{i}}{\partial X^{\alpha} \partial X^{\mu}} \frac{\partial X^{\eta}}{\partial x^{k}} \frac{\partial x^{j}}{\partial X^{\xi}}\right] \\
& =\tilde{R}_{\eta}^{\alpha[\xi \mu]}\left[\Gamma^{j}{ }_{\alpha \xi} \frac{\partial^{2} X^{\eta}}{\partial x^{k} \partial x^{\nu}} \frac{\partial x^{k}}{\partial X^{j}} \frac{\partial x^{\nu}}{\partial X^{\mu}}+\gamma^{k}{ }_{i j} \frac{\partial^{2} x^{i}}{\partial X^{\alpha} \partial X^{\mu}} \frac{\partial X^{\eta}}{\partial x^{k}} \frac{\partial x^{j}}{\partial X^{\xi}}\right] \\
& =\tilde{R}_{\eta}^{\alpha[\xi \mu]}\left[\Gamma_{\alpha \xi}^{j}\left(\gamma_{k \nu}^{i} \frac{\partial X^{\eta}}{\partial x^{i}} \frac{\partial x^{k}}{\partial X^{j}} \frac{\partial x^{\nu}}{\partial X^{\mu}}-\Gamma_{j \mu}^{\eta}\right)+\gamma_{i j}^{k}\left(\Gamma_{\alpha \mu}^{a} \frac{\partial x^{i}}{\partial X^{a}}-\gamma_{a b}^{i} \frac{\partial x^{a}}{\partial X^{\alpha}} \frac{\partial x^{b}}{\partial X^{\mu}}\right) \frac{\partial X^{\eta}}{\partial x^{k}} \frac{\partial x^{j}}{\partial X^{\xi}}\right] \\
& =\tilde{R}_{\eta}{ }^{\alpha[\xi \mu]}\left(-\Gamma_{\alpha \xi}^{i} \Gamma_{i \mu}^{\eta}-\gamma_{a b}^{i} \gamma_{i j}^{k} \frac{\partial x^{a}}{\partial X^{\alpha}} \frac{\partial x^{b}}{\partial X^{\mu}} \frac{\partial X^{\eta}}{\partial x^{k}} \frac{\partial x^{j}}{\partial X^{\xi}}+\Gamma_{\alpha \xi}^{j}{ }_{\alpha{ }_{k \nu}}^{i} \frac{\partial X^{\eta}}{\partial x^{i}} \frac{\partial x^{k}}{\partial X^{j}} \frac{\partial x^{\nu}}{\partial X^{\mu}}+\Gamma_{\alpha \mu}^{j} \gamma_{k \nu}^{i} \frac{\partial X^{\eta}}{\partial x^{i}} \frac{\partial x^{k}}{\partial X^{j}} \frac{\partial x^{\nu}}{\partial X^{\xi}}\right) \\
& =-\tilde{R}_{\eta}{ }^{\alpha[\xi \mu]} \Gamma_{\alpha \xi}^{i} \Gamma_{i \mu}^{\eta}+\gamma_{a b}^{i} \gamma_{i j}^{k} \tilde{R}_{\eta}{ }^{\alpha[\xi \mu]} \frac{\partial x^{a}}{\partial X^{\alpha}} \frac{\partial x^{b}}{\partial X^{\xi}} \frac{\partial X^{\eta}}{\partial x^{k}} \frac{\partial x^{j}}{\partial X^{\mu}} \\
& =-\tilde{R}_{\eta}{ }^{\alpha[\xi \mu]} \Gamma_{\alpha \xi}^{i} \Gamma^{\eta}{ }_{i \mu}+\tilde{r}_{k}{ }^{a[b j]} \gamma_{a b}^{i} \gamma^{k}{ }_{i j}=-\tilde{R}_{\eta}{ }^{\alpha[\xi \mu]} \Gamma_{\alpha \xi^{i}} \Gamma^{\eta}{ }_{i \mu}+\tilde{r}_{\eta}{ }^{\alpha[\xi \mu]} \gamma_{\alpha \xi}^{i} \gamma^{\eta}{ }_{i \mu} \\
& =-\frac{1}{2} \tilde{R}_{\eta}{ }^{\alpha \xi \mu}\left(\Gamma^{i}{ }_{\alpha \xi} \Gamma^{\eta}{ }_{i \mu}-\Gamma_{\alpha \mu}^{i} \Gamma^{\eta}{ }_{i \xi}\right)+\frac{1}{2} \tilde{r}_{\eta}{ }^{\alpha \xi \mu}\left(\gamma_{\alpha \xi}^{i} \gamma_{i \mu}^{\eta}-\gamma_{\alpha \mu}^{i} \gamma_{i \xi}^{\eta}\right) \text {. }
\end{aligned}
$$

The two mixed terms in $\Gamma, \gamma$ cancel each other due to the skew-symmetry of $\tilde{R}_{\eta}^{\alpha[\xi \mu]}$ in $\xi, \mu$.

The contribution of (26) emerging from the symmetric part $\tilde{R}_{\eta}{ }^{\alpha(\xi \mu)}$ can be expressed in terms of the derivatives of the connection coefficients, whose transformation rule is

$$
\begin{aligned}
\frac{\partial \Gamma_{\alpha \xi}^{\eta}}{\partial X^{\kappa}} \frac{\partial X^{\kappa}}{\partial x^{\nu}}= & \frac{\partial \gamma^{k}{ }_{i j}}{\partial x^{\nu}} \frac{\partial X^{\eta}}{\partial x^{k}} \frac{\partial x^{i}}{\partial X^{\alpha}} \frac{\partial x^{j}}{\partial X^{\xi}} \\
& +\gamma^{k}{ }_{i j} \frac{\partial}{\partial x^{\nu}}\left(\frac{\partial X^{\eta}}{\partial x^{k}} \frac{\partial x^{i}}{\partial X^{\alpha}} \frac{\partial x^{j}}{\partial X^{\xi}}\right)
\end{aligned}
$$

$$
+\frac{\partial}{\partial x^{\nu}}\left(\frac{\partial X^{\eta}}{\partial x^{k}} \frac{\partial^{2} x^{k}}{\partial X^{\alpha} \partial X^{\xi}}\right)
$$

Thus

$$
\begin{array}{r}
\tilde{R}_{\eta}{ }^{\alpha(\xi \mu)} \frac{\partial x^{\nu}}{\partial X^{\mu}}\left[\gamma^{k}{ }_{i j} \frac{\partial}{\partial x^{\nu}}\left(\frac{\partial X^{\eta}}{\partial x^{k}} \frac{\partial x^{i}}{\partial X^{\alpha}} \frac{\partial x^{j}}{\partial X^{\xi}}\right)\right. \\
\left.+\frac{\partial}{\partial x^{\nu}}\left(\frac{\partial X^{\eta}}{\partial x^{k}} \frac{\partial^{2} x^{k}}{\partial X^{\alpha} \partial X^{\xi}}\right)\right]
\end{array}
$$




$$
\begin{aligned}
& =\tilde{R}_{\eta}{ }^{\alpha(\xi \mu)} \frac{\partial x^{\nu}}{\partial X^{\mu}}\left(\frac{\partial \Gamma^{\eta}{ }_{\alpha \xi}}{\partial X^{\kappa}} \frac{\partial X^{\kappa}}{\partial x^{\nu}}-\frac{\partial \gamma^{k}{ }_{i j}}{\partial x^{\nu}} \frac{\partial X^{\eta}}{\partial x^{k}} \frac{\partial x^{i}}{\partial X^{\alpha}} \frac{\partial x^{j}}{\partial X^{\xi}}\right) \\
& =\tilde{R}_{\eta}{ }^{\alpha(\xi \mu)} \frac{\partial \Gamma^{\eta}{ }_{\alpha \xi}}{\partial X^{\mu}}-\tilde{r}_{k}{ }^{i(j \nu)} \frac{\partial \gamma^{k}{ }_{i j}}{\partial x^{\nu}} \\
& =\tilde{R}_{\eta}{ }^{\alpha(\xi \mu)} \frac{\partial \Gamma_{\alpha \xi}^{\eta}}{\partial X^{\mu}}-\tilde{r}_{\eta}{ }^{\alpha(\xi \mu)} \frac{\partial \gamma^{\eta}{ }_{\alpha \xi}}{\partial x^{\mu}} \\
& =\frac{1}{2} \tilde{R}_{\eta}{ }^{\alpha \xi \mu}\left(\frac{\partial \Gamma^{\eta}{ }_{\alpha \xi}}{\partial X^{\mu}}+\frac{\partial \Gamma^{\eta}{ }_{\alpha \mu}}{\partial X^{\xi}}\right)-\frac{1}{2} \tilde{r}_{\eta}{ }^{\alpha \xi \mu}\left(\frac{\partial \gamma^{\eta}{ }_{\alpha \xi}}{\partial x^{\mu}}+\frac{\partial \gamma^{\eta}{ }_{\alpha \mu}}{\partial x^{\xi}}\right) .
\end{aligned}
$$

The total transformation rule (D1) expressed in terms of connection coefficients is then

$$
\begin{aligned}
& \tilde{T}_{\alpha}{ }^{\beta} \frac{\partial X^{\alpha}}{\partial y^{\beta}}-\tilde{t}_{\alpha}{ }^{\beta} \frac{\partial x^{\alpha}}{\partial y^{\beta}} \\
&= \frac{1}{2} \tilde{R}_{\eta}{ }^{\alpha \xi \mu}\left(\frac{\partial \Gamma^{\eta}{ }_{\alpha \xi}}{\partial X^{\mu}}+\frac{\partial \Gamma^{\eta}{ }_{\alpha \mu}}{\partial X^{\xi}}-\Gamma_{\alpha \xi}^{i}{ }_{\alpha{ }^{\prime}{ }_{i \mu}}+\Gamma_{\alpha \mu}^{i}{ }_{\alpha \mu} \Gamma_{i \xi}^{\eta}\right) \\
&-\frac{1}{2} \tilde{r}_{\eta}{ }^{\alpha \xi \mu}\left(\frac{\partial \gamma^{\eta}{ }_{\alpha \xi}}{\partial x^{\mu}}+\frac{\partial \gamma^{\eta}{ }_{\alpha \mu}}{\partial x^{\xi}}-\gamma_{\alpha \xi}^{i} \gamma_{i \mu}^{\eta}+\gamma_{\alpha \mu}^{i} \gamma_{i \xi}^{\eta}\right) . \\
& \hline
\end{aligned}
$$

\section{Appendix E: EXPLICIT CALCULATION OF THE EULER-LAGRANGE EQUATION (38)}

The form-invariant extended Lagrangian describing the source-free space-time dynamics is given by

$$
\begin{aligned}
& \mathcal{L}_{\mathrm{e}}\left(\gamma^{\eta}{ }_{\alpha \xi}, \frac{\partial \gamma^{\eta}{ }_{\alpha \xi}}{\partial x^{\nu}}, \frac{\partial x^{\mu}}{\partial y^{\nu}}\right) \\
& =-\frac{1}{4} g_{\kappa \eta} g^{\beta \alpha} g^{\lambda \xi} g^{\zeta \tau} r^{\kappa}{ }_{\beta \lambda \zeta} r^{\eta}{ }_{\alpha \xi \tau} \operatorname{det} \Lambda
\end{aligned}
$$

with $g_{\kappa \eta}$ denoting the covariant metric tensor and $g^{\beta \alpha}$ its contravariant counterpart. In this description, the curvature tensor $r_{\beta \lambda \zeta}^{\kappa}$-as defined by Eq. (30) — only depends on the connection coefficients and their space-time derivatives. Thus, only the metric tensors and $\operatorname{det} \Lambda$ have derivatives with respect to the space-time coefficients $\partial x^{\mu} / \partial y^{\nu}$. These derivatives were worked out explicitly with Eqs. (A5), (A6), and (A2). The derivative of $\mathcal{L}_{\mathrm{e}}$ with respect to the space-time coefficients follows as

$$
\begin{aligned}
& \frac{\partial \mathcal{L}_{\mathrm{e}}}{\partial\left(\frac{\partial x^{\mu}}{\partial y^{\nu}}\right)}=-\frac{1}{4} r^{\kappa}{ }_{\beta \lambda \zeta} r_{\alpha \xi \tau}^{\eta} \operatorname{det} \Lambda\left[\frac{\partial g_{\kappa \eta}(y)}{\partial\left(\frac{\partial x^{\mu}}{\partial y^{\nu}}\right)} g^{\beta \alpha} g^{\lambda \xi} g^{\zeta \tau}+\frac{\partial g^{\beta \alpha}(y)}{\partial\left(\frac{\partial x^{\mu}}{\partial y^{\nu}}\right)} g_{\kappa \eta} g^{\lambda \xi} g^{\zeta \tau}\right. \\
& \left.+\frac{\partial g^{\lambda \xi}(y)}{\partial\left(\frac{\partial x^{\mu}}{\partial y^{\nu}}\right)} g_{\kappa \eta} g^{\beta \alpha} g^{\zeta \tau}+\frac{\partial g^{\zeta \tau}(y)}{\partial\left(\frac{\partial x^{\mu}}{\partial y^{\nu}}\right)} g_{\kappa \eta} g^{\beta \alpha} g^{\lambda \xi}+\frac{\partial y^{\nu}}{\partial x^{\mu}} g_{\kappa \eta} g^{\beta \alpha} g^{\lambda \xi} g^{\zeta \tau}\right] \\
& =-\frac{1}{4} r_{\beta \lambda \zeta}^{\kappa} r_{\alpha \xi \tau}^{\eta} \operatorname{det} \Lambda \frac{\partial y^{j}}{\partial x^{\mu}}\left[\left(\delta_{\kappa}^{\nu} g_{j \eta}+\delta_{\eta}^{\nu} g_{\kappa j}\right) g^{\beta \alpha} g^{\lambda \xi} g^{\zeta \tau}\right. \\
& -\left(\delta_{j}^{\beta} g^{\nu \alpha}+\delta_{j}^{\alpha} g^{\beta \nu}\right) g_{\kappa \eta} g^{\lambda \xi} g^{\zeta \tau}-\left(\delta_{j}^{\lambda} g^{\nu \xi}+\delta_{j}^{\xi} g^{\lambda \nu}\right) g_{\kappa \eta} g^{\beta \alpha} g^{\zeta \tau} \\
& \left.-\left(\delta_{j}^{\zeta} g^{\nu \tau}+\delta_{j}^{\tau} g^{\zeta \nu}\right) g_{\kappa \eta} g^{\beta \alpha} g^{\lambda \xi}+\delta_{j}^{\nu} g_{\kappa \eta} g^{\beta \alpha} g^{\lambda \xi} g^{\zeta \tau}\right] \\
& =\frac{1}{4} \operatorname{det} \Lambda \frac{\partial y^{j}}{\partial x^{\mu}}\left(-r^{\nu}{ }_{\beta \lambda \zeta} r_{\alpha \xi \tau}^{\eta} g_{j \eta} g^{\beta \alpha} g^{\lambda \xi} g^{\zeta \tau}-r_{\beta \lambda \zeta}^{\kappa}{ }^{\nu}{ }_{\alpha \xi \tau} g_{\kappa j} g^{\beta \alpha} g^{\lambda \xi} g^{\zeta \tau}\right. \\
& +r^{\kappa}{ }_{j \lambda \zeta} r^{\eta}{ }_{\alpha \xi \tau} g_{\kappa \eta} g^{\nu \alpha} g^{\lambda \xi} g^{\zeta \tau}+r^{\kappa}{ }_{\beta \lambda \zeta} r^{\eta}{ }_{j \xi \tau} g_{\kappa \eta} g^{\beta \nu} g^{\lambda \xi} g^{\zeta \tau} \\
& +r_{\beta j \zeta}^{\kappa} r_{\alpha \xi \tau}^{\eta} g_{\kappa \eta} g^{\beta \alpha} g^{\nu \xi} g^{\zeta \tau}+r_{\beta \lambda \zeta}^{\kappa} r_{\alpha j \tau}^{\eta} g_{\kappa \eta} g^{\beta \alpha} g^{\lambda \nu} g^{\zeta \tau} \\
& +r_{\beta \lambda j}^{\kappa} r_{\alpha \xi \tau}^{\eta} g_{\kappa \eta} g^{\beta \alpha} g^{\lambda \xi} g^{\nu \tau}+r_{\beta \lambda \zeta}^{\kappa} r_{\alpha \xi j}^{\eta} g_{\kappa \eta} g^{\beta \alpha} g^{\lambda \xi} g^{\zeta \nu} \\
& \left.-\delta_{j}^{\nu} r_{\beta \lambda \zeta}^{\kappa} r_{\alpha \xi \tau}^{\eta} g_{\kappa \eta} g^{\beta \alpha} g^{\lambda \xi} g^{\zeta \tau}\right) \\
& =\frac{1}{4} \operatorname{det} \Lambda \frac{\partial y^{j}}{\partial x^{\mu}}\left(-2 r^{\nu \alpha \xi \tau} r_{j \alpha \xi \tau}+2 r^{\eta \nu \xi \tau} r_{\eta j \xi \tau}+2 r^{\eta \alpha \nu \tau} r_{\eta \alpha j \tau}+2 r^{\eta \alpha \xi \nu} r_{\eta \alpha \xi j}-\delta_{j}^{\nu} r^{\eta \alpha \xi \tau} r_{\eta \alpha \xi \tau}\right) \\
& =\left.\left(-\frac{1}{2} r^{\nu \alpha \xi \tau} r_{j \alpha \xi \tau}+\frac{1}{2} r^{\eta \nu \xi \tau} r_{\eta j \xi \tau}+r^{\eta \alpha \xi \nu} r_{\eta \alpha \xi j}-\frac{1}{4} \delta_{j}^{\nu} r^{\eta \alpha \xi \tau} r_{\eta \alpha \xi \tau}\right)\right|_{y} \frac{\partial y^{j}}{\partial x^{\mu}} \operatorname{det} \Lambda \\
& =\left.\left(-\frac{1}{2} r^{j \alpha \xi \tau} r_{\mu \alpha \xi \tau}+\frac{1}{2} r^{\eta j \xi \tau} r_{\eta \mu \xi \tau}+r^{\eta \alpha \xi j} r_{\eta \alpha \xi \mu}-\frac{1}{4} \delta_{\mu}^{j} r^{\eta \alpha \xi \tau} r_{\eta \alpha \xi \tau}\right)\right|_{x} \frac{\partial y^{\nu}}{\partial x^{j}} \operatorname{det} \Lambda .
\end{aligned}
$$

The first two terms cancel under the precondition that $r^{\nu \alpha \xi \tau}$ is skew-symmetric also in its first index pair. This is ensured if a metric is present ([15, p 324]), which is assumed in the actual context. The final result is thus

$$
\frac{\partial \mathcal{L}_{\mathrm{e}}}{\partial\left(\frac{\partial x^{\mu}}{\partial y^{\nu}}\right)}=\left(r^{\eta \alpha \xi j} r_{\eta \alpha \xi \mu}-\frac{1}{4} \delta_{\mu}^{j} r^{\eta \alpha \xi \beta} r_{\eta \alpha \xi \beta}\right) \frac{\partial y^{\nu}}{\partial x^{j}} \operatorname{det} \Lambda .
$$


[1] J. Struckmeier, J. Phys. G: Nucl. Part. Phys. 40, 015007 (2013).

[2] J. Struckmeier and H. Reichau,

[8] J. Struckmeier, J. Phys. A: Math. Gen. 38, 1257 (2005).

[9] J. Struckmeier, Int. J. Mod. Phys. E 18, 79 (2009).

FIAS Interdisciplinary Science Series (Springer, New York, 2013) p. 367 , p. 367.

[3] L. H. Ryder, Introduction to General Relativity (Cambridge University Press, Cambridge, England, 2009).

[4] T.-P. Cheng and L.-F. Li, Gauge Theory of Elementary Particle Physics (Oxford University Press, New York, 2000).

[5] F. Hehl, P. von der Heyde, G. Kerlick, and J. Nester, Rev. Mod. Phys. 48, 393 (1976).

[6] A. Palatini, Rend. Circ. Mat. Palermo 43, 203 (1919).

[7] J. V. José and E. J. Saletan, Classical Dynamics (Cambridge University Press, Cambridge, 1998).

J. Cosmol. Astropart. Phys. 11, 008 (2008)

[11] G. Stephenson, Il Nuovo Cimento 9, 263 (1958).

[12] C. Lanczos, Ann. Math. 39, 842 (1938).

[13] K. S. Stelle, Phys. Rev. D 16, 953 (1977).

[14] A. Einstein, "Private letter to H. Weyl," ETH Zürich Library, Archives and Estates (1918).

[15] C. W. Misner, K. S. Thorne, and J. A. Wheeler, Gravitation (W. H. Freeman and Company, New York, 1973). 